\title{
Priors for Genotyping Polyploids
}

\author{
David Gerard ${ }^{1}$ and Luís Felipe Ventorim Ferrão² \\ ${ }^{1}$ Department of Mathematics and Statistics, American University, Washington, DC, 20016, USA \\ ${ }^{2}$ Horticultural Sciences Department, University of Florida, Gainesville, FL, 32611, USA
}

\begin{abstract}
Motivation: Empirical Bayes techniques to genotype polyploid organisms usually either (i) assume technical artifacts are known a priori or (ii) estimate technical artifacts simultaneously with the prior genotype distribution. Case (i) is unappealing as it places the onus on the researcher to estimate these artifacts, or to ensure that there are no systematic biases in the data. However, as we demonstrate with a few empirical examples, case (ii) makes choosing the class of prior genotype distributions extremely important. Choosing a class that is either too flexible or too restrictive results in poor genotyping performance.
\end{abstract}

Results: We propose two classes of prior genotype distributions that are of intermediate levels of flexibility: the class of proportional normal distributions and the class of unimodal distributions. We provide a complete characterization of and optimization details for the class of unimodal distributions. We demonstrate, using both simulated and real data, that using these classes results in superior genotyping performance.

Availability and Implementation: Genotyping methods that use these priors are implemented in the updog $\mathrm{R}$ package available on the Comprehensive $\mathrm{R}$ Archive Network: https: //cran.r-project.org/package=updog. All code needed to reproduce the results of this paper is available on GitHub: https://github.com/dcgerard/reproduce_prior_sims.

Contact: dgerard@american.edu

\section{Introduction}

Because of their importance in agriculture [Udall and Wendel, 2006], evolution [Soltis et al., 2014], and biodiversity [Soltis and Soltis, 2000], scientists are increasingly interested in studying the genomic landscape of polyploid organisms (organisms with more than two copies of their genome). In these studies, researchers usually first estimate the number of copies of each allele (dosage) at given polymorphic loci in a sample. These dosages may then be fed into downstream analyses such as genome-wide association studies [Rosyara et al., 2016, Ferrão et al., 2018, Benevenuto et al., 2019], genomic prediction [Endelman et al., 2018, Amadeu et al., 2019, de Bem Oliveira et al., 2019, Lara et al., 2019], and genetic mapping [Shirasawa et al., 2017, Ferreira et al., 2019]. To determine allele dosage, researchers first measure the reference and alternative counts of small reads from

Keywords and phrases: Allele dosage, GBS, RAD-seq, SNP, sequencing, empirical Bayes. 
some sequencing technology [Baird et al., 2008, Elshire et al., 2011]. Based on these read counts, they then infer the dosage of reference and alternative alleles using statistical methods.

Due to constraints in sequencing depth, many genotyping methods incorporate empirical Bayes techniques to borrow strength between individuals [Voorrips et al., 2011, Serang et al., 2012, Maruki and Lynch, 2017, Blischak et al., 2018, Gerard et al., 2018, Clark et al., 2019]. These methods posit some class of prior distributions for the possible genotypes, select a distribution among this class using maximum marginal likelihood, and incorporate this prior genotype distribution in a Bayesian inference scheme to derive a posterior genotype distribution from which genotype calls are made. Adaptively estimating the prior distribution allows methods to automatically gauge the level of prior information in the data and use this prior information to improve genotyping.

Besides the genotype of the individual, there are usually other, more technical, aspects of the data that should be taken into consideration during allele dosage inference, including sequencing error rate, allele bias, and overdispersion [Gerard et al., 2018]. A principled empirical Bayes approach would integrate over the uncertainty in these other parameters prior to estimating the genotype prior. However, computation is a major concern as there are often tens or hundreds of thousands of SNPs to genotype in a single dataset, and even increases of seconds can make a genotyping method untenable for applied researchers. Thus, for computational reasons, genotyping methods either assume these parameters are known a priori [Maruki and Lynch, 2017, Blischak et al., 2018, Clark et al., 2019] or estimate them simultaneously with the prior genotype distribution [Voorrips et al., 2011, Gerard et al., 2018].

Assuming these parameters are known a priori is unappealing as it prohibits adapting to the features present in the data. However, using the second strategy of simultaneously estimating technical features with the genotype prior makes the choice of the class of priors of vital importance. To demonstrate this, we present a genotype plot [Gerard et al., 2018] of a single SNP from the autotetraploid potato data from Uitdewilligen et al. [2013] in panel A of Figure 1. A genotype plot contains the reference counts of an individual on the $y$-axis and the alternative counts on the $x$-axis. Individuals with a dosage of four reference alleles would lie near the $x=0$ vertical line, while individuals with a dosage of zero reference alleles would lie near the $y=0$ horizontal line. The plot seems to display two clusters of individuals, which would indicate to a researcher that there is a group of potatoes with a genotype of four reference alleles and a group of potatoes with a genotype of three reference alleles. However, when we fit updog (the software implementation of the empirical Bayes genotyping method described in Gerard et al. [2018]) to these data, we obtain unreasonable genotype estimates when we either allow the class of priors to be anything over genotypes $\{0,1,2,3,4\}$ (panel B of Figure 1 ) or we constrain the prior to be the discrete uniform distribution over genotypes $\{0,1,2,3,4\}$ (panel $\mathrm{C}$ of Figure 1). These results generalize to other datasets (Supplementary Figure S1). (As a preview, panel D of Figure 1 demonstrates that using one of our proposed classes of prior distributions results in intuitive genotype estimates).

This indicates that using a class of priors that is either too flexible or too restrictive can result in poor genotyping. In the case of the discrete uniform prior, updog effectively tries to estimate the allele bias and sequencing error to make the empirical genotype distribution as close to a discrete uniform as possible. As the real genotype distribution is not discrete uniform, this results in extreme estimates of the allele bias and unreasonable genotype estimates. In the case of the general prior, since we do not have a data-adaptive prior distribution on the bias, there is little loss in marginal likelihood for having an extreme allele bias. And so the bias parameter is free to vary as long as it increases the marginal likelihood. Indeed, if we include a slight penalty on the bias parameter, 

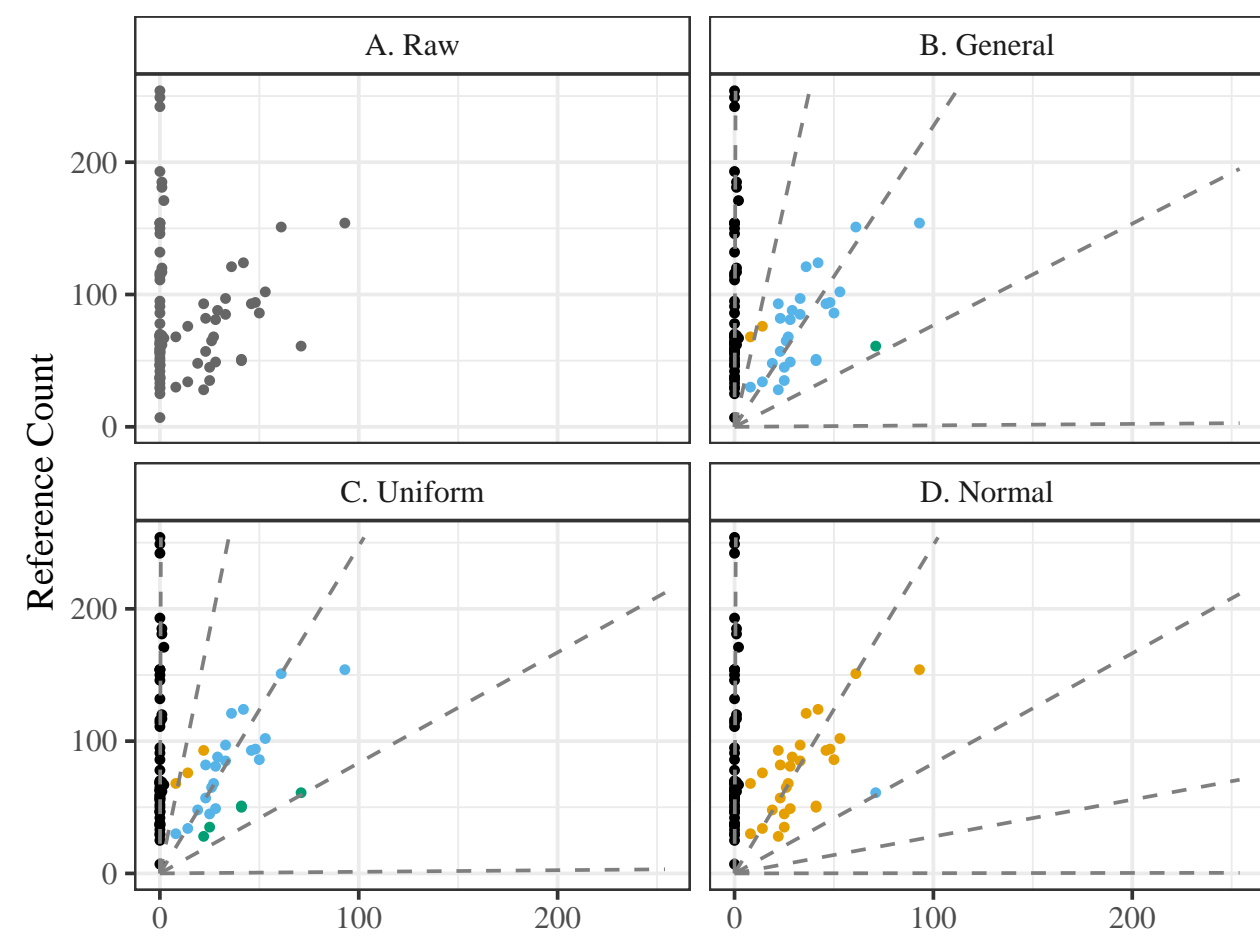

\section{Genotype}

- 4

- 3

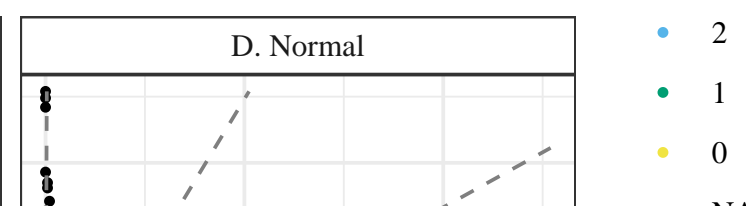

Alternative Count

Figure 1: The number of reference reads ( $y$-axis) versus the number of alternative reads ( $x$-axis) from 84 autotetraploid potatoes for a SNP from Uitdewilligen et al. [2013]. The raw unannotated data are presented in panel (A). The other panels are color-coded by estimated genotype according to updog fits using either (B) the general class of priors, $(\mathrm{C})$ the discrete uniform prior distribution, or (D) the class of proportional normal priors. The dashed lines in panels (B) through (D) are the estimated expected counts, and are functions of the estimated allele bias and estimated sequencing error rate [see Gerard et al., 2018]. Only the proportional normal class of priors provides intuitive genotyping.

the general class of prior distributions results in reasonable genotype estimates (Supplementary Figure S2). These results indicate that having too flexible a class of prior distributions can result in unstable genotype estimates; while having too constrained a class of prior distributions can result in the likelihood parameters adapting to the prior distribution instead of having the prior distribution improve our estimates of the genotypes.

In this paper, we propose two classes of prior distributions for genotyping polyploids which are designed to have intermediate levels of flexibility (Section 2). One is the class of discrete unimodal distributions, for which we provide a complete characterization. The other is the class of distributions whose probability masses are proportional to a normal density. We provide optimization details for these classes, along with other classes of priors used in the literature. We demonstrate, through simulation and on real data, that these two classes, and in particular the proportional normal class, is the most robust to varying genotype distributions (Section 3). The proportional normal prior is the default in the updog software available on the Comprehensive $\mathrm{R}$ Archive Network: https://cran.r-project.org/package=updog. 


\section{Methods}

We now provide a brief description of the model and genotyping procedure we study. We assume that all inference is done one SNP at a time (though see the discussion in Section 4). Let $x_{i}$ be the reference read-counts for individual $i$, let $n_{i}$ be the total number of reads for individual $i$, let $K$ be the ploidy of the species under study, let $y_{i} \in\{0,1, \ldots, K\}$ be the (unknown) genotype for individual $i$, let $\boldsymbol{\eta}$ be a vector of likelihood-specific parameters (such as the allele bias, sequencing error rate, or overdispersion), and let $\boldsymbol{\theta}$ be a vector of prior-specific parameters. Different values of $\boldsymbol{\theta}$ index the class of allowable genotype distributions. Let $f\left(x_{i} \mid y_{i}, n_{i}, \boldsymbol{\eta}\right)$ denote the likelihood of $x_{i}$. This could, for example, be the likelihood from Voorrips et al. [2011] or Gerard et al. [2018], but we allow it to be more general. Let $f\left(y_{i} \mid \boldsymbol{\theta}\right)$ denote the prior probability of genotype $y_{i}$. We suppose that the inference strategy consists of the following two steps: First, estimate the likelihood- and prior-specific parameters by maximum marginal likelihood:

$$
(\hat{\boldsymbol{\eta}}, \hat{\boldsymbol{\theta}})=\underset{\boldsymbol{\eta}, \boldsymbol{\theta}}{\arg \max } \sum_{i} \log \left(\sum_{y_{i}=0}^{K} f\left(y_{i} \mid \boldsymbol{\theta}\right) f\left(x_{i} \mid y_{i}, n_{i}, \boldsymbol{\eta}\right)\right)
$$

and second, derive the posterior distribution of the genotypes for each individual:

$$
f\left(y_{i} \mid x_{i}, n_{i}, \hat{\boldsymbol{\eta}}, \hat{\boldsymbol{\theta}}\right)=\frac{f\left(y_{i} \mid \hat{\boldsymbol{\theta}}\right) f\left(x_{i} \mid y_{i}, n_{i}, \hat{\boldsymbol{\eta}}\right)}{\sum_{y_{i}=0}^{K} f\left(y_{i} \mid \hat{\boldsymbol{\theta}}\right) f\left(x_{i} \mid y_{i}, n_{i}, \hat{\boldsymbol{\eta}}\right)} .
$$

These posterior probabilities are then used to make genotype calls. Deriving a general-purpose expectation-maximization algorithm [Dempster et al., 1977] to obtain $\hat{\boldsymbol{\eta}}$ and $\hat{\boldsymbol{\theta}}$ is not difficult (Supplementary Section S2.1), though special optimization considerations are needed for some of the priors we discuss in this section (Supplementary Sections S2.1 and S2.2).

Prior distributions that have been used in the past (though not necessarily in the above framework) include:

1. The discrete uniform distribution [McKenna et al., 2010]:

$$
f\left(y_{i}=k\right)=1 /(K+1), \text { where } \boldsymbol{\theta} \text { is the null vector. }
$$

2. The distribution of autopolyploid sibling genotypes [Serang et al., 2012]:

$$
\begin{gathered}
f\left(y_{i}=k \mid \ell_{1}, \ell_{2}\right)=\sum_{i=0}^{k} \operatorname{HG}\left(i, K / 2 \mid \ell_{1}, K\right) \operatorname{HG}\left(k-i, K / 2 \mid \ell_{2}, K\right), \text { where } \\
\operatorname{HG}(a, b \mid c, d)=\frac{\left(\begin{array}{l}
c \\
a
\end{array}\right)\left(\begin{array}{l}
d-c \\
b-a
\end{array}\right)}{\left(\begin{array}{l}
d \\
b
\end{array}\right)}, \boldsymbol{\theta}=\left(\ell_{1}, \ell_{2}\right), \text { and } \ell_{1}, \ell_{2} \in\{0,1, \ldots, K\} .
\end{gathered}
$$

Equation (4) is merely a convolution of two hypergeometric distributions. The $\ell_{1}$ and $\ell_{2}$ parameters are the genotypes of the two parents. We will denote this distribution as the "F1 distribution" as it results from an F1 cross of autopolyploid individuals. 
3. The binomial distribution [Martin et al., 2010, Li, 2011]:

$$
f\left(y_{i}=k \mid \alpha\right)=\left(\begin{array}{c}
K \\
k
\end{array}\right) \alpha^{k}(1-\alpha)^{K-k}, \text { where } \boldsymbol{\theta}=\alpha, \text { for } \alpha \in[0,1] .
$$

The $\alpha$ parameter is the allele frequency of the reference allele. The binomial distribution results by assuming the individuals are in Hardy-Weinberg equilibrium.

4. The beta-binomial distribution [Blischak et al., 2018]:

$$
f\left(y_{i}=k \mid \alpha, \gamma\right)=\left(\begin{array}{c}
K \\
k
\end{array}\right) \frac{\mathrm{B}\left(k+\alpha \frac{1-\gamma}{\gamma}, K-k+(1-\alpha) \frac{1-\gamma}{\gamma}\right)}{\mathrm{B}\left(\alpha \frac{1-\gamma}{\gamma},(1-\alpha) \frac{1-\gamma}{\gamma}\right)}, \text { where } \boldsymbol{\theta}=(\alpha, \gamma), \text { for } \alpha, \gamma \in[0,1]
$$

where $\mathrm{B}(a, b)$ represents the beta function with parameters $a$ and $b$. The $\alpha$ parameter is the allele frequency of the reference allele, while $\gamma$ is an overdispersion parameter. This is slightly different than the prior used in Blischak et al. [2018] as there the overdispersion parameter is individual-specific, while in (6) it is locus-specific.

5. The general categorical distribution [DePristo et al., 2011, Li, 2011]:

$$
f\left(y_{i}=k \mid \pi_{0}, \ldots, \pi_{K}\right)=\pi_{k}, \text { where } \boldsymbol{\theta}=\left(\pi_{0}, \ldots, \pi_{K}\right) \text { such that } \pi_{k} \in[0,1] \text { and } \sum_{k} \pi_{k}=1 .
$$

There are many issues with these previously used priors. The issues with the discrete uniform and general distributions were already noted in Section 1. The F1 and binomial distributions are only applicable in specific situations. The F1 distribution can only be applied if the individuals are known siblings, the binomial distribution relies on the strong assumptions of Hardy-Weinberg equilibrium [Crow, 1988]. Though the beta-binomial distribution can account for overdispersion, it is unable to account for underdispersion, which may be caused by, e.g., unobserved relatedness (for example, the F1 distribution is generally less dispersed than a binomial, and hence a beta-binomial).

In order to account for both over- and underdispersion, in our first proposal we model genotype probabilities as being proportional to a normal density:

$$
f\left(k \mid \mu, \sigma^{2}\right)=\frac{\mathrm{N}\left(k \mid \mu, \sigma^{2}\right)}{\sum_{k=0}^{K} \mathrm{~N}\left(k \mid \mu, \sigma^{2}\right)}, \text { where } \boldsymbol{\theta}=\left(\mu, \sigma^{2}\right) \in[0, K] \times \mathbb{R}^{+},
$$

where $\mathrm{N}\left(\cdot \mid \mu, \sigma^{2}\right)$ represents the normal density with mean $\mu$ and variance $\sigma^{2}$. This distribution, though somewhat ad hoc, can approximate very well distributions from the classes of binomial, beta-binomial, and F1 distributions (Supplementary Figure S3). One can thus essentially consider it a general class that encompasses as special cases these classes of distributions. Varying $\sigma^{2}$ allows for the prior distribution to accommodate both over- and underdispersion. We call this the "proportional normal distribution".

Motivated by the fact that most of the prior distributions previously proposed in the literature are unimodal, our second proposal is to model genotype probabilities within the flexible class of unimodal probability mass functions. In Supplementary Section S1 we exactly characterize this class of distributions using mixtures of discrete uniform distributions. This idea was motivated by 
the work of Stephens [2016] who approximated continuous densities using mixtures of continuous uniform distributions. Our characterization lends itself to a convex optimization problem during the M-step of the EM algorithm (Supplementary Section S2.1). But rather than use standard convex optimization techniques, we found it faster to develop a weighted EM scheme to optimize over the class of unimodal distributions (Supplementary Section S2.2).

All priors explored in this paper are summarized in Supplementary Table S2.

\section{Results}

\subsection{Simulation results}

In this section we compare, through simulation, the seven different classes of prior distributions from Section 2. Our strategy was to generate genotypes under seven different genotype distributions, where each genotype distribution was designed to be favorable to one of the classes of prior distributions. The discrete uniform, binomial (Hardy-Weinberg), beta-binomial, and F1 favorable genotype distributions were all generated according to their assumed priors. For the genotype distribution favorable to the proportional normal prior, we generated genotypes according to the proportional normal distribution with a small variance since we motivated this distribution by the need to account for underdispersion. For the unimodal favorable genotype distribution, we allowed genotype probabilities to decline linearly from $K$ to 0 since none of the other more structured prior classes have such linear declines. Finally, for the genotype distribution favorable to the general prior, we specified non-unimodal genotype proportions, which none of the other priors should be able to accommodate. The specific genotype distributions are listed in Supplementary Section S3 and are graphically represented in Supplementary Figure S6.

Given individual genotypes, we generated sequencing data according to the likelihood in Gerard et al. [2018], setting the sequencing error rate to 0.001, varying the overdispersion parameter over $\{0,0.005,0.01\}$ (where larger values indicate greater dispersion), and varying the allele bias parameter over $\{1,0.75,0.5\}$ (where values further from 1 indicate greater bias). Each repetition, we generated thirty hexaploid individuals at a sequencing depth of 100 reads. For each unique combination of allele bias, overdispersion, and genotype distribution, we generated 500 datasets. For each dataset, we fit updog assuming one of the seven prior classes described in Section 2.

The results under conditions of no allele bias and no overdispersion are presented in Figures 2 and 3. The results under varying levels of bias and overdispersion are presented in Supplementary Figures S7-S27. Figure 2 plots the proportion of individuals genotyped correctly (with larger values being better) while Figure 3 plots the difference between the estimated proportion of individuals misclassified and the actual proportion of individuals misclassified (with values closer to 0 being better). In Figures 2 and 3, we see that each class of prior distributions performs best when the genotype distribution is designed to be favorable to it. Except for the classes of proportional normal distributions and unimodal distributions, every class performs very poorly under some genotype distribution. However, the class of unimodal distributions performs poorly under some genotype distributions under larger levels of bias and overdispersion (Supplementary Figures S8, S11, S15, and S18). The class of proportional normal distributions only performs poorly under the (unrealistic) general-favorable genotype distribution with large levels of bias and overdispersion (Supplementary Figures S13 and S20). Only the class of proportional normal priors performs adequately when the gentoypes are very underdispersed (Supplementary Figures S11 and S18). Es- 

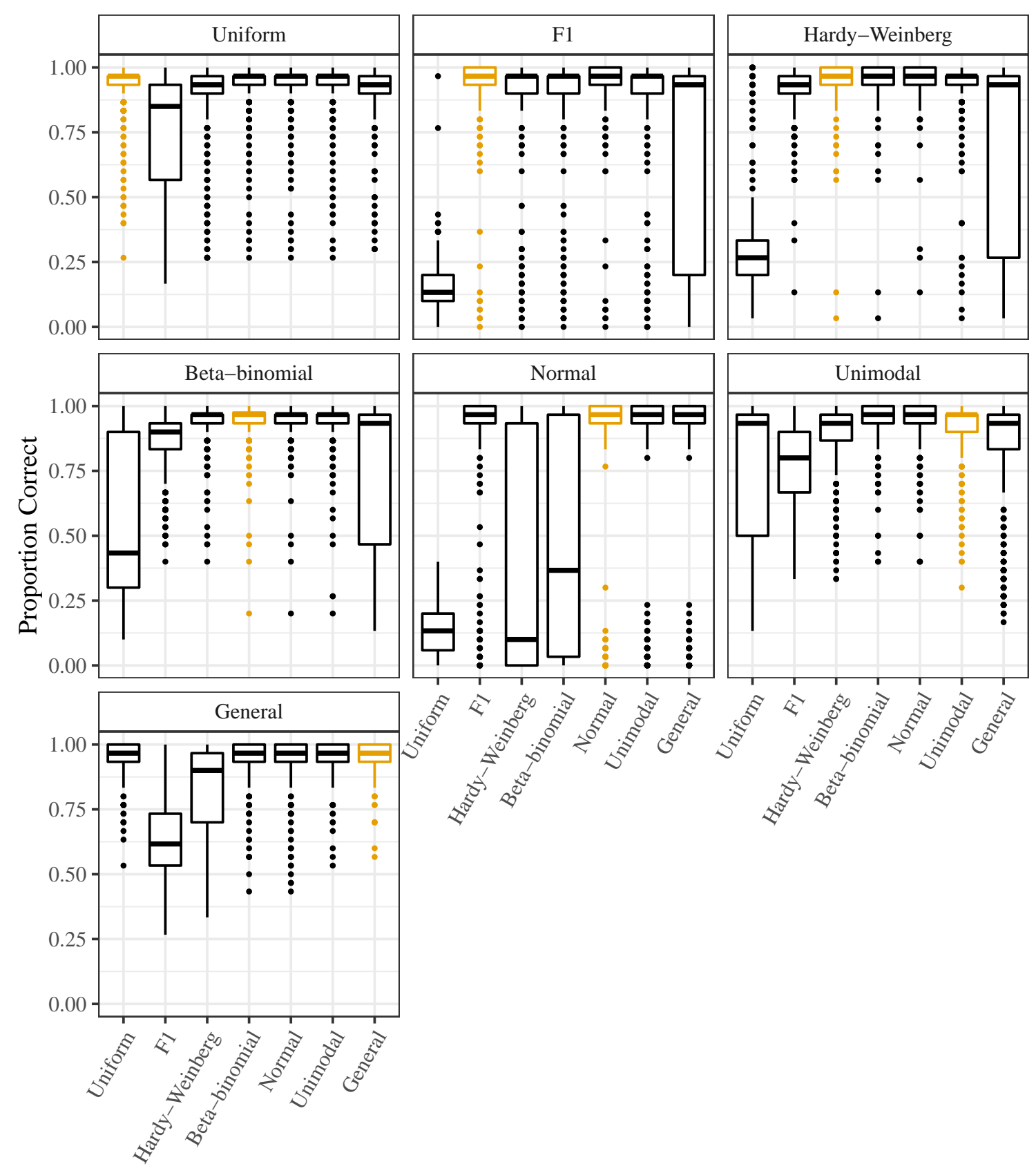

\section{Assumed Prior}

Figure 2: Proportion of individuals genotyped correctly ( $y$-axis) stratified by the assumed class of prior distributions $(x$-axis) used in an updog fit. The facets index the distributions used to generate the genotypes, with each genotype distribution designed to be favorable to one of the classes of prior distributions (orange). The distributions are ordered roughly from least flexible (the discrete uniform) to most flexible (the general class). The read counts were generated assuming no allele bias and no overdispersion. 
timates of the allele bias when using the class of proportional normal priors appear to be unbiased (Supplementary Figures S23-S27). These results indicate that the class of proportional normal distributions performs the best under a wide variety of settings.

\subsection{Sweet potato results}

In this section, we compare the seven classes of prior distributions discussed in Section 2 using the data from Shirasawa et al. [2017]. The hexaploid sweet potatoes in these data are known siblings, resulting from a single generation of selfing (an S1 cross). Thus, the class of genotype distributions is known to be F1 (4) with the constraint that $\ell_{1}=\ell_{2}$ [Gerard et al., 2018], which we will simply call "S1". We fit updog, using all seven prior classes in Section 2 as well as the S1 prior class, to the 1000 SNPs with mean read-depth closest to 100. We ran updog only on a sample of twenty individuals, as having fewer individuals makes the class of prior distributions most important. For each SNP, we calculated the Euclidean distances between all pairs of posterior mean genotypes resulting from the eight different fitted prior classes. We also calculated the Hamming distances (divided by the number of individuals) between the posterior mode genotypes resulting from the eight different fitted prior classes.

The results are presented in Figure 4. As the genotype distribution is known to belong to the $\mathrm{S} 1$ class, we will consider its genotypes a proxy for the true genotypes. Thus, prior classes that are closest to S1 (in terms of either Euclidean distance from posterior mean genotypes, or Hamming distance from posterior mode genotypes) perform the best. We see in Figure 4 that F1 performs the best. This is expected since S1 is a small subclass of the class of F1 distributions. The second best performance comes from the class of proportional normal priors. Thus, on real data, we see the gains attained by using the class of proportional normal priors. Mean distances between the results of all methods are presented in Supplementary Figures S28 and S29.

\section{Discussion}

In this paper, we reviewed the classes of prior distributions used to genotype polyploids, found these classes either too flexible or too restrictive, and proposed two new classes of priors of intermediate levels of flexibility. This required us to characterize the class of discrete unimodal distributions using mixtures of discrete uniform distributions. We then demonstrated, through simulation, that our proposals work better under the genotyping inference scheme in updog. In particular, we recommend to use by default the class of proportional normal distributions as it resulted in robust performance under different gentoype distributions. The proportional normal prior is the default in the updog software available on the Comprehensive R Archive Network: https://cran.r-project. org/package=updog. Our classes of priors are also more generally applicable to other inference schemes.

Our priors are appropriate defaults for a wide variety of genotyping scenarios. For example, breeding populations might exhibit violations in Mendelian segregation proportions (4) due to double reduction [Stift et al., 2010], preferential pairing [Voorrips and Maliepaard, 2012], or resulting from an allele being semi-lethal. Additionally, unstructured populations might exhibit violations from Hardy-Weinberg equilibrium (5) due to selection or non-random mating [Crow, 1988]. Our proportional normal and unimodal classes of priors may be used in all of these scenarios without explicitly modeling these processes. 
Here, we only analyzed one SNP at a time, though some recent approaches have also attempted to borrow strength between SNPs [Blischak et al., 2018, Clark et al., 2019]. In particular, Clark et al. [2019] tries to account for linkage disequilibrium (LD) between SNPs. However, their method, though intuitive, is somewhat ad hoc. Generalizing our proportional normal prior would allow for a more principled approach to accounting for LD by simply letting the genotype distribution across multiple SNPs be proportional to a multivariate normal. To account for the high-dimensionality present due to the large number of SNPs, one could impose strong structural constraints on the covariance matrix, such as letting it decay exponentially according to recombination rate [Wen and Stephens, 2010].

\section{Data availability}

All methods are implemented in the updog software available on the Comprehensive $\mathrm{R}$ Archive Network: https://cran.r-project.org/package=updog. All code and instructions to reproduce the results of this paper are available on GitHub: https://github.com/dcgerard/reproduce prior_sims.

\section{Acknowledgments}

All graphics were made using ggplot2 [Wickham, 2016] in the R statistical language [R Core Team, 2019].

We thank the College of Arts and Sciences at American University for providing a travel grant from the Mellon Fund. This allowed the authors to meet and collaborate on this manuscript. 


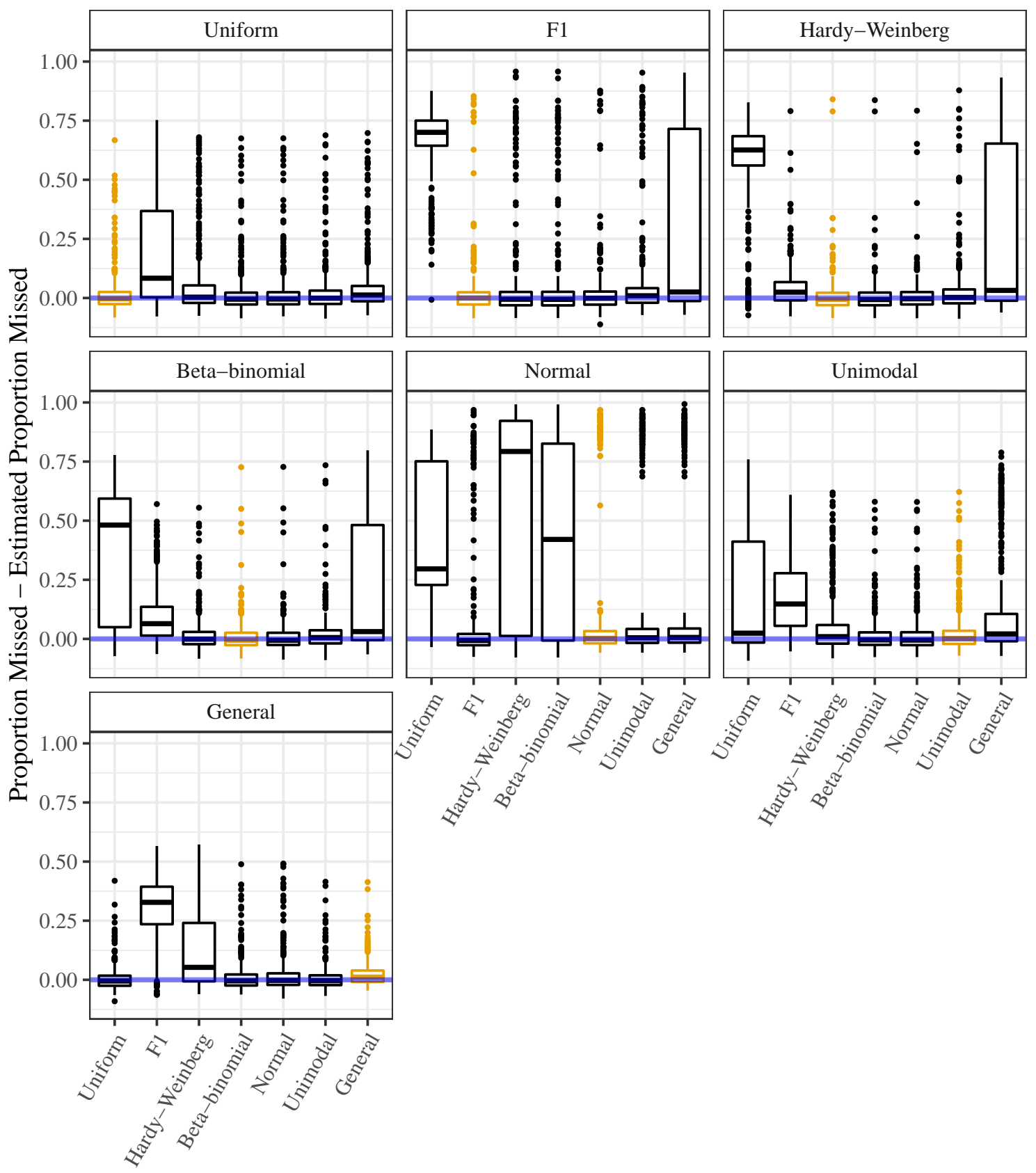

Assumed Prior

Figure 3: Estimated proportion of individuals incorrectly genotyped subtracted from the actual proportion of individuals incorrectly genotyped ( $y$-axis) stratified by the assumed class of prior distributions ( $x$-axis) used in an updog fit. The horizontal line (at $y=0$ ) indicates unbiased estimation of the misclassification error rate. The facets index the distributions used to generate the genotypes, with each genotype distribution designed to be favorable to one of the classes of prior distributions (orange). The distributions are ordered roughly from least flexible (the discrete uniform) to most flexible (general). The read counts were generated assuming no allele bias and no overdispersion. 


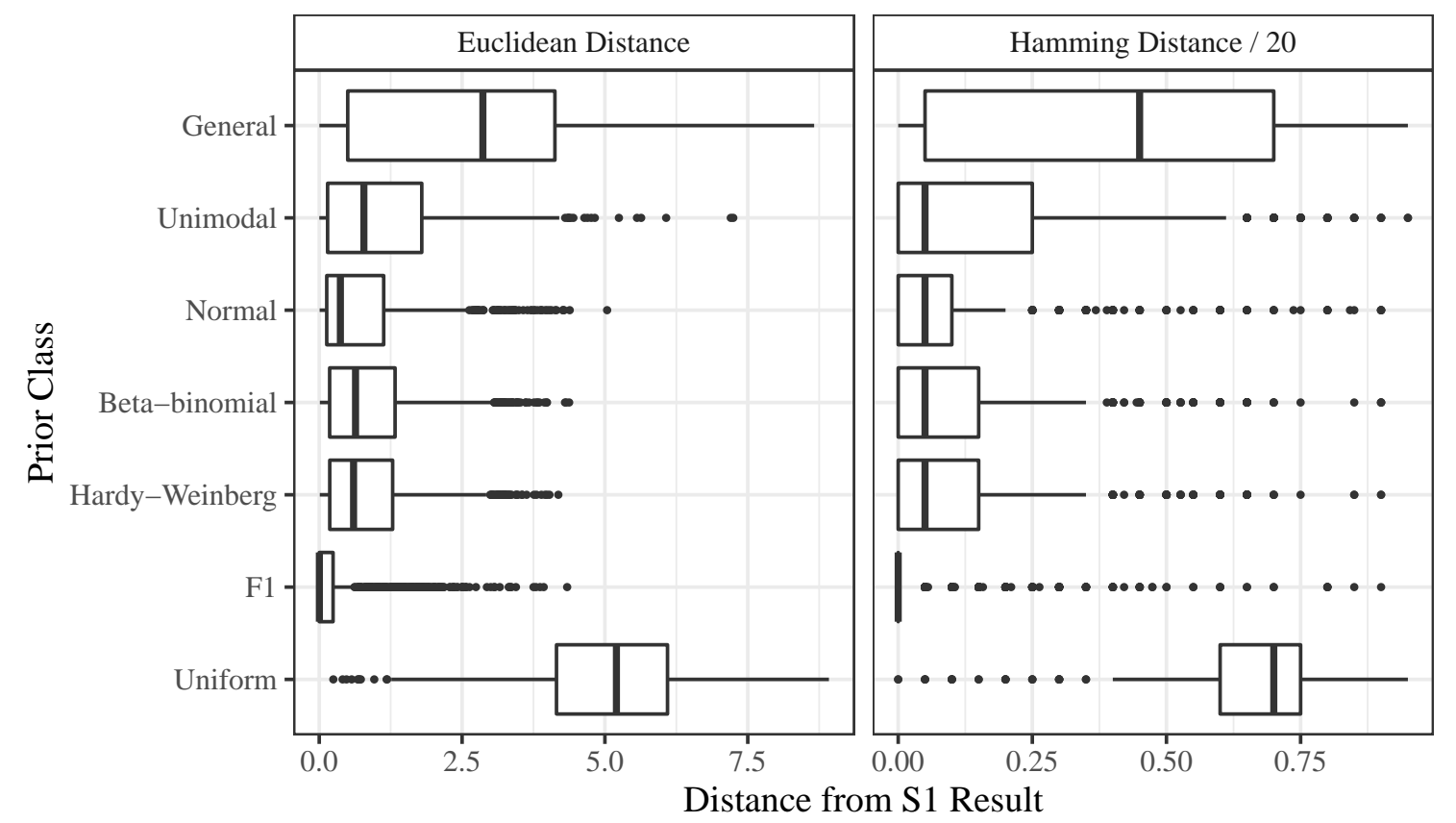

Figure 4: Boxplots of distances ( $x$-axis) stratified by prior class ( $y$-axis). The left facet contains the Euclidean distances between posterior means when either using the S1 prior or the specified prior on the $y$-axis. The right facet contains the proportion of individuals genotyped differently when either using the $\mathrm{S} 1$ prior or the specified prior on the $y$-axis. Distances closer to 0 indicate superior performance. 


\section{S1 Characterizing the class of discrete unimodal distributions}

Theorem 1. Let $\mathcal{F}(a, b)$ denote the set of integers between $a$ and $b$. That is,

$$
\mathcal{F}(a, b): \mathbb{R} \times \mathbb{R} \rightarrow 2^{\mathbb{Z}}, \quad \mathcal{F}(a, b)=\{x \in \mathbb{Z}: x \in[\min (a, b), \max (a, b)]\} .
$$

Then a probability mass function $f(\cdot)$ with support on $\{0,1, \ldots, K\}$ is unimodal if and only if there exists an $a \in(-1, K+1) \backslash\{0,1, \ldots, K\}$ and $\left\{\pi_{0}, \pi_{1}, \ldots, \pi_{K}\right\}$ with $\pi_{i} \in[0,1]$ and $\sum_{i=0}^{K} \pi_{i}=1$ such that

$$
f(k)=\sum_{i=0}^{K} \pi_{i} \frac{1(k \in \mathcal{F}(a, i))}{|\mathcal{F}(a, i)|},
$$

where $|\mathcal{A}|$ denotes the number of elements in a set $\mathcal{A}$ and $1(\cdot)$ is the indicator function.

Proof. We will start by proving the "if" part. Suppose (10) holds. We must then prove three things: (i) $f(\cdot)$ is a probability mass function, (ii) if $k_{1}<k_{2}<a$ then $f\left(k_{1}\right) \leq f\left(k_{2}\right)$, and (iii) if $k_{1}>k_{2}>a$ then $f\left(k_{1}\right) \leq f\left(k_{2}\right)$. Note that $f(k)$ is non-negative for all $k$ since the summands in (10) are always non-negative. We also have

$$
\begin{aligned}
\sum_{k=0}^{K} f(k) & =\sum_{k=0}^{K} \sum_{i=0}^{K} \pi_{i} \frac{1(k \in \mathcal{F}(a, i))}{|\mathcal{F}(a, i)|} \\
& =\sum_{i=0}^{K} \frac{\pi_{i}}{|\mathcal{F}(a, i)|} \sum_{k=0}^{K} 1(k \in \mathcal{F}(a, i)) \\
& =\sum_{i=0}^{K} \frac{\pi_{i}}{|\mathcal{F}(a, i)|}|\mathcal{F}(a, i)| \\
& =\sum_{i=0}^{K} \pi_{i} \\
& =1
\end{aligned}
$$

Thus, $f(\cdot)$ is a valid probability mass function. Now let $k_{1}<k_{2}<a$. Then

$$
\begin{aligned}
f\left(k_{2}\right)-f\left(k_{1}\right) & =\sum_{i=0}^{K} \pi_{i} \frac{1\left(k_{2} \in \mathcal{F}(a, i)\right)}{|\mathcal{F}(a, i)|}-\sum_{i=0}^{K} \pi_{i} \frac{1\left(k_{1} \in \mathcal{F}(a, i)\right)}{|\mathcal{F}(a, i)|} \\
& =\sum_{i=0}^{K} \frac{\pi_{i}}{|\mathcal{F}(a, i)|}\left(1\left(k_{2} \in \mathcal{F}(a, i)\right)-1\left(k_{1} \in \mathcal{F}(a, i)\right)\right) .
\end{aligned}
$$

Note that if $k_{1} \in \mathcal{F}(a, i)$ then $k_{2} \in \mathcal{F}(a, i)$, so $1\left(k_{2} \in \mathcal{F}(a, i)\right) \geq 1\left(k_{1} \in \mathcal{F}(a, i)\right)$. Thus, the summands in (17) are all non-negative, which implies that $f\left(k_{2}\right) \geq f\left(k_{1}\right)$. The proof for when $k_{1}>k_{2}>a$ is similar. We have thus proved the "if" part.

We now prove the "only if" part. Suppose $f(\cdot)$ is a unimodal probability mass function with support on $\{0,1, \ldots, K\}$. Then there exists an $a \in(-1, K+1) \backslash\{0,1, \ldots, K\}$ such that $k_{1}<$ $k_{2}<a$ implies $f\left(k_{1}\right) \leq f\left(k_{2}\right)$ and $k_{1}>k_{2}>a$ implies that $f\left(k_{1}\right) \leq f\left(k_{2}\right)$. For example, let 
$j \in\{0,1, \ldots, K\}$ be any mode of $f(\cdot)$, then choose $a=j+\epsilon$ for any $\epsilon<1 /(K+1)$. Now set

$$
\pi_{i}= \begin{cases}(f(i)-f(i-1))|\mathcal{F}(a, i)| & \text { if } i<a, \\ (f(i)-f(i+1))|\mathcal{F}(a, i)| & \text { if } i>a .\end{cases}
$$

We must now show three things: (i) the definition of the $\pi_{i}$ 's used in (18) results in equality (10), (ii) the $\pi_{i}$ 's are all between 0 and 1 , and (iii) the $\pi_{i}$ 's sum to 1 . First, suppose that $k<a$. Then

$$
\begin{aligned}
\sum_{i=0}^{K} \pi_{i} \frac{1(k \in \mathcal{F}(a, i))}{|\mathcal{F}(a, i)|} & =\sum_{i=0}^{k} \frac{\pi_{i}}{|\mathcal{F}(a, i)|} \\
& =\sum_{i=0}^{k} \frac{(f(i)-f(i-1))|\mathcal{F}(a, i)|}{|\mathcal{F}(a, i)|} \\
& =\sum_{i=0}^{k}(f(i)-f(i-1)) \\
& =f(k)
\end{aligned}
$$

Equation (19) follows since $1(k \in \mathcal{F}(a, i))$ is 0 for $i>k$ and is 1 for $i \leq k$. Equation (22) follows from (21) by a telescoping argument and noting that $f(-1)=0$. The argument for when $k>a$ is similar. Thus, we have that (10) holds. By the unimodality of $f(\cdot)$, the $\pi_{i}$ 's in (18) are non-negative. Thus, it suffices to show that they sum to one. We have

$$
\begin{aligned}
\sum_{i=0}^{K} \pi_{i}= & \sum_{i<a} \pi_{i}+\sum_{i>a} \pi_{i} \\
= & \sum_{i<a}(f(i)-f(i-1))|\mathcal{F}(a, i)|+\sum_{i>a}(f(i)-f(i+1))|\mathcal{F}(a, i)| \\
= & \sum_{i<a} f(i)|\mathcal{F}(a, i)|-\sum_{i<a} f(i-1)|\mathcal{F}(a, i)|+\sum_{i>a} f(i)|\mathcal{F}(a, i)|-\sum_{i>a} f(i+1)|\mathcal{F}(a, i)| \\
= & \sum_{i<a} f(i)|\mathcal{F}(a, i)|-\sum_{i<a-1} f(i)|\mathcal{F}(a, i+1)|+\sum_{i>a} f(i)|\mathcal{F}(a, i)|-\sum_{i>a+1} f(i)|\mathcal{F}(a, i-1)| \\
= & \sum_{i<a-1} f(i)(|\mathcal{F}(a, i)|-|\mathcal{F}(a, i+1)|)+f(\lfloor a\rfloor)|\mathcal{F}(a,\lfloor a\rfloor)| \\
& +\sum_{i>a+1} f(i)(|\mathcal{F}(a, i)|-|\mathcal{F}(a, i-1)|)+f(\lceil a\rceil)|\mathcal{F}(a,\lceil a\rceil)| \\
= & \sum_{i<a-1} f(i)+f(\lfloor a\rfloor)+\sum_{i>a+1} f(i)+f(\lceil a\rceil) \\
= & \sum_{i} f(i) \\
= & 1
\end{aligned}
$$

Equation (28) follows from the fact that $|\mathcal{F}(a, i)|-|\mathcal{F}(a, i+1)|=1$ for $i<a,|\mathcal{F}(a, i)|-|\mathcal{F}(a, i-1)|=$ 1 for $i>a$, and $|\mathcal{F}(a,\lfloor a\rfloor)|=|\mathcal{F}(a,\lceil a\rceil)|=1$. 


\section{S2 Optimization details}

\section{S2.1 EM derivation}

We will now derive a general-purpose EM algorithm to solve (1). Let $\boldsymbol{z}_{i}$ be a 1-of- $(K+1)$ vector indicating the genotype for individual $i$. Then the Complete log-likelihood of the $\boldsymbol{z}_{i}$ 's and $x_{i}$ 's is:

$$
\sum_{k=0}^{K} \sum_{i} z_{i k} \log f(k \mid \boldsymbol{\theta})+\sum_{k=0}^{K} \sum_{i} z_{i k} \log f\left(x_{i} \mid k, n_{i}, \boldsymbol{\eta}\right) .
$$

Using standard calculations, the posterior expectation of $z_{i k}$ is

$$
w_{i k}:=E\left[z_{i k} \mid x_{i}, \boldsymbol{\theta}, \boldsymbol{\eta}\right]=\frac{f\left(x_{i} \mid k, n_{i}, \boldsymbol{\eta}\right) f(k \mid \boldsymbol{\theta})}{\sum_{k} f\left(x_{i} \mid k, n_{i}, \boldsymbol{\eta}\right) f(k \mid \boldsymbol{\theta})},
$$

And the expected complete log-likelihood is

$$
\sum_{k} w_{k} \log f(k \mid \boldsymbol{\theta})+\sum_{k} \sum_{i} w_{i k} \log f\left(x_{i} \mid k, n_{i}, \boldsymbol{\eta}\right),
$$

where $w_{k}:=\sum_{i} w_{i k}$. Thus, the E-step of the EM algorithm consists of calculating the posterior expectations from (32). The M-step consists of two independent optimization problems, one for the likelihood-specific parameters and one for the prior-specific parameters:

$$
\begin{aligned}
& \boldsymbol{\theta}=\underset{\boldsymbol{\theta}}{\arg \max } \sum_{k} w_{k} \log f(k \mid \boldsymbol{\theta}), \\
& \boldsymbol{\eta}=\underset{\boldsymbol{\eta}}{\arg \max } \sum_{k} \sum_{i} w_{i k} \log f\left(x_{i} \mid k, n_{i}, \boldsymbol{\eta}\right) .
\end{aligned}
$$

Since the E-step and the optimization in (35) is the same no matter the choice of class of priors, we focus on the optimization in (34).

We will now derive the optimization updates for the each of the classes of prior distributions discussed in Section 2. There is no prior optimization step for the discrete uniform distribution (3). For the beta-binomial (6) and proportional normal (8) distributions, we merely use gradient ascent to solve (34). For the binomial distribution (5), there exists a closed-form solution for update (34):

$$
\alpha=\frac{\sum_{k=0}^{K} k w_{k}}{K \sum_{k=0}^{K} w_{k}}
$$

We also have a closed-form solution for the update with the general prior (7):

$$
\pi_{k}=\frac{w_{k}}{\sum_{k=0}^{K} w_{k}} .
$$

Note that $\sum_{k} w_{k}$ in (36) and (37) is merely the number of individuals in the sample.

A naive update for the F1 distribution (4) would simply iterate over the possible levels of 
$\left(\ell_{1}, \ell_{2}\right) \in\{0,1, \ldots, K\} \times\{0,1, \ldots, K\}$. However, this approach causes the resulting EM-algorithm to get stuck at certain levels of $\left(\ell_{1}, \ell_{2}\right)$. To see this, recall that the prior optimization problem during the M-step is

$$
\max _{\ell_{1}, \ell_{2}} \sum_{k} w_{k} \log f\left(k \mid \ell_{1}, \ell_{2}\right)
$$

If $w_{i}$ is non-zero during the current M-step, then moving to an $\left(\ell_{1}, \ell_{2}\right)$ such that $f\left(i \mid \ell_{1}, \ell_{2}\right)=0$ would result in a likelihood of $-\infty$. Also, if the current values of $\left(\ell_{1}, \ell_{2}\right)$ result in a non-zero $f\left(i \mid \ell_{1}, \ell_{2}\right)$, then (32) implies that $w_{i}$ can never become 0 during the E-step. This is major issue for the EM algorithm since the F1 distribution contains many exact zero probabilities, effectively inhibiting any exploration of $\left(\ell_{1}, \ell_{2}\right)$. To remedy this, we introduce a small discrete uniform mixing component:

$$
f\left(k \mid \ell_{1}, \ell_{2}, \gamma\right)=(1-\gamma) f\left(k \mid \ell_{1}, \ell_{2}\right)+\gamma /(K+1)
$$

for some $\gamma \in(0,1)$. A principled approach would be to take $\gamma$ to 0 during the EM-optimization. However, we have found it sufficient to simply set $\gamma$ to a small value $\left(10^{-3}\right.$ by default).

A simpler approach to optimization using the F1 distribution would be to simply run $K(K+1) / 2$ different EM-algorithms - one for each possible combination of $\left(\ell_{1}, \ell_{2}\right)$. However, we have found the approach of a single EM algorithm using a modified F1 distribution (39) much faster in practice and yields the same results. For example, on a $2.6 \mathrm{GHz}$ quad-core PC running Linux with $32 \mathrm{~GB}$ of memory, running updog with the single EM approach with a discrete uniform mixing component took 2.6 seconds, 2.0 seconds, and 1.9 seconds on the three example SNPs used in Gerard et al. [2018]. On these same SNPs, running updog with the multiple EM approach took 15.7 seconds, 14.4 seconds, and 14.0 seconds. They yielded nearly the exact same posterior mean genotypes except in one individual in one SNP (Supplementary Figure S4).

To update the parameters from the class of unimodal distributions, we first assume that the "center" value of $a$ is known (see Theorem 1 ). There exists a finite number of $a$ values over which the likelihood varies which may be iterated over each M-step. Then let $\ell_{i k}:=1(k \in \mathcal{F}(a, i)) /|\mathcal{F}(a, i)|$. The prior optimization problem during the M-step then becomes

$$
\max _{\pi_{0}, \pi_{1}, \ldots, \pi_{K}} \sum_{k=0}^{K} w_{k} \log \left(\sum_{i=0}^{K} \pi_{i} \ell_{i k}\right) .
$$

Equation (40) is a convex optimization problem and can be solved using standard interior-point methods. However, we found that in this particular problem (with a small value of $K$ ), we can obtain minor gains in performance by using a weighted EM algorithm to solve (40) (Supplementary Section S2.2).

\section{S2.2 Weighted EM derivation for the class of unimodal distributions}

In this section, we describe a weighted EM algorithm to solve (40). This is an alternative optimization scheme to standard interior point methods. For the moment, suppose the $w_{k}$ 's in (40) are 
natural numbers. Then we can equivalently write (40) as

$$
\max _{\pi_{0}, \pi_{1}, \ldots, \pi_{K}} \sum_{k^{*}=1}^{w} \log \left(\sum_{i=0}^{K} \pi_{i} \ell_{i k^{*}}^{*}\right),
$$

where

$$
\begin{aligned}
w & :=\sum_{k=0}^{K} w_{k}, \text { and } \\
\sum_{k^{*}=1}^{w} 1\left(\ell_{i k^{*}}^{*}=\ell_{i k} \forall i\right) & =w_{k} .
\end{aligned}
$$

That is, for each $k$ we make $w_{k}$ copies of $\left(\ell_{0 k}, \ell_{1 k}, \ldots \ell_{K k}\right)$. Equation (41) is equivalent to equation (S.2.6) of the Supplementary Material in Stephens [2016], and so the EM algorithm in Stephens [2016] may be used to update (41):

$$
\begin{aligned}
n_{i} & =\sum_{k^{*}=1}^{w} \frac{\pi_{i} \ell_{i k^{*}}^{*}}{\sum_{i=0}^{K} \pi_{i} \ell_{i k^{*}}^{*}}, \\
\pi_{i} & =\frac{n_{i}}{\sum_{i=0}^{K} n_{i}}
\end{aligned}
$$

To solve (40) using the original parameter values, note that (44) is equivalent to

$$
n_{i}=\sum_{k=0}^{K} w_{k} \frac{\pi_{i} \ell_{i k}}{\sum_{i=0}^{K} \pi_{i} \ell_{i k}} .
$$

Since the update for the $\pi_{i}$ 's is invariant to scalar multiplication of the $w_{k}$ 's, we may update (40) for non-integer $w_{k}$ 's using (46) followed by (45).

The $\ell_{i k}$ 's contain many exact zeros, which can cause convergence problem for the weighted EM algorithm. This can be remedied by placing a small Dirichlet prior on the $\pi_{i}$ 's (with the same concentration parameter placed on each $\pi_{i}$ ). The resulting weighted EM algorithm is

$$
\begin{aligned}
n_{i} & =\sum_{k=0}^{K} w_{k} \frac{\pi_{i} \ell_{i k}}{\sum_{i=0}^{K} \pi_{i} \ell_{i k}}+\lambda, \\
\pi_{i} & =\frac{n_{i}}{\sum_{i=0}^{K} n_{i}} .
\end{aligned}
$$

By default, we choose $\lambda=10^{-6}$.

We found that using the weighted EM algorithm performed faster in this scenario than using interior point methods. On a $2.6 \mathrm{GHz}$ quad-core PC running Linux with $32 \mathrm{~GB}$ of memory, running updog with the weighted EM approach took 50.3 seconds, 34.4 seconds, 24.0 and seconds on the three example SNPs used in Gerard et al. [2018]. On these same SNPs, running updog with interior point methods (using the CVXR R package from Fu et al. [2017]) took 163.1 seconds, 87.8 seconds, and 66.2 seconds. The posterior mean genotypes were almost exactly the same using both 
optimization approaches (Supplementary Figure S5).

\section{S3 Genotype distributions used in the simulation study}

In Section 3, genotypes were generated under the following seven conditions. Before each distribution, we list for which prior this genotype distribution was intended to be favorable.

1. Hardy-Weinberg Favorable: $\operatorname{Pr}(k)=\operatorname{Binomial}(k \mid K, 0.75)$,

2. Beta-Binomial Favorable: $\operatorname{Pr}(k)=\mathrm{BB}(k \mid K, 0.75,0.1)$,

3. Proportional Normal Favorable: $\operatorname{Pr}(k) \propto N\left(k \mid 4.5,0.5^{2}\right)$,

4. Unimodal Favorable: $\operatorname{Pr}(k)=k / \sum_{k=0}^{K} k$,

5. F1 Favorable: $\operatorname{Pr}(k)$ was derived from one parent with 4 copies and one parent with 5 copies of reference allele,

6. General Favorable: $\boldsymbol{\pi}=(1,4,1,4,1,4,8) / 23$, and

7. Discrete Uniform Favorable: $\operatorname{Pr}(k)=1 /(K+1)$.

These genotype distributions (except the discrete uniform distribution) are plotted in Supplementary Figure S6. 


\section{S4 Supplementary figures}

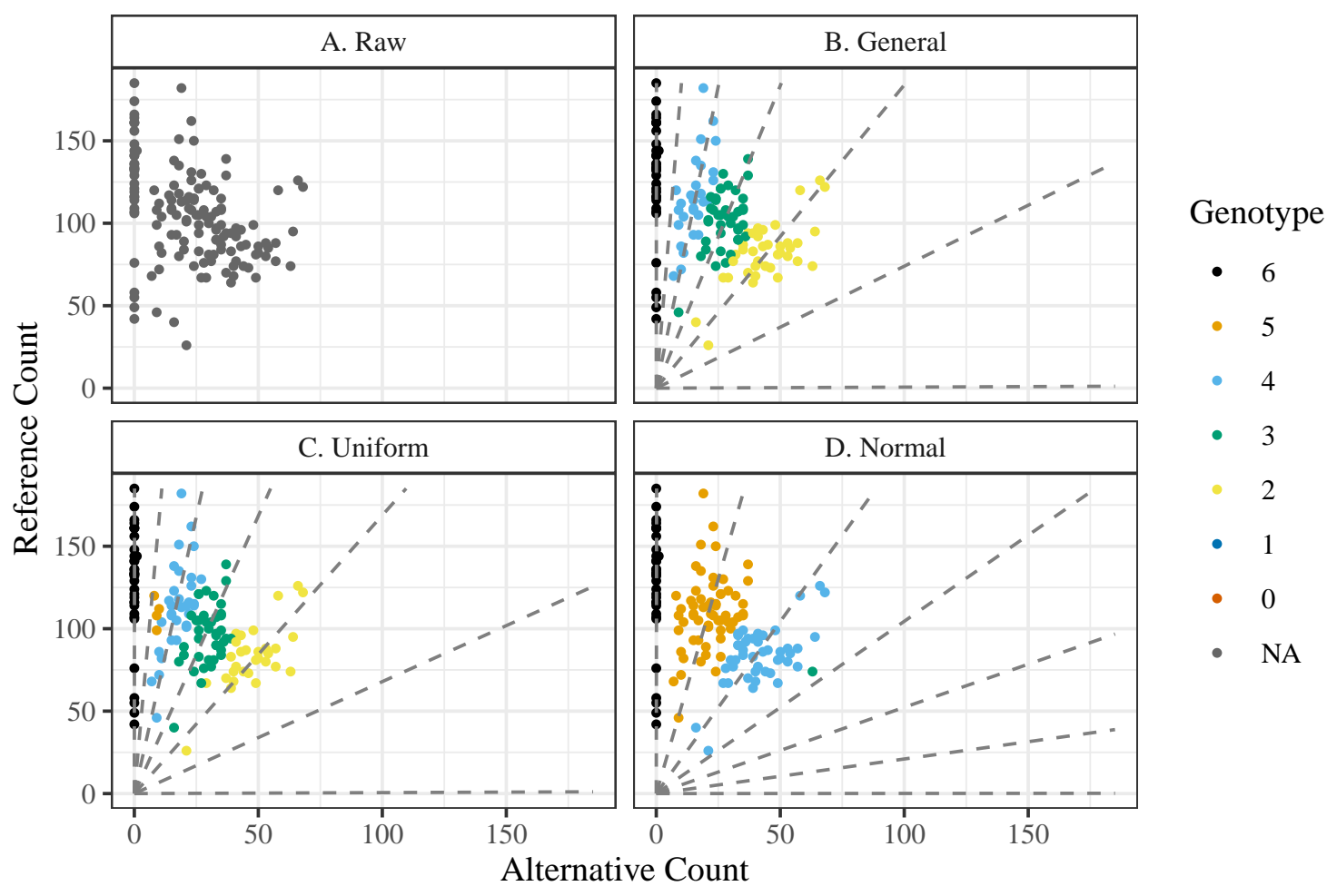

Figure S1: The number of reference reads ( $y$-axis) versus the number of alternative reads ( $x$-axis) for a SNP from 143 sweet potatoes from Shirasawa et al. [2017]. The raw unannotated data are presented in panel (A). The other panels are color-coded by estimated genotype according to updog fits using either (B) the general class of priors, (C) the discrete uniform prior distribution, or (D) the class of proportional normal priors. The dashed lines in panels (B) through (D) are the estimated expected counts, and are functions of the estimated allele bias and estimated sequencing error rate [see Gerard et al., 2018]. These individuals are known to be siblings resulting from an S1 cross. Given this knowledge, only the proportional normal class of priors provides intuitive genotyping.

\begin{tabular}{|c|c|c|c|}
\hline Distribution & Distribution Parameters & Normal Mean & Normal SD \\
\hline Uniform & $\cdot$ & 3 & $\infty$ \\
\hline F1 & $\ell_{1}=1$ and $\ell_{2}=2$ & 1.5 & 0.8 \\
\hline Hardy-Weinberg (Binomial) & $\alpha=0.25$ & 1.4 & 1.2 \\
\hline Beta-binomial & $\alpha=0.25$ and $\gamma=0.01$ & 0.8 & 1.8 \\
\hline
\end{tabular}

Table S1: Parameter values for the proportional normal distributions displayed in Supplementary Figure S3. 


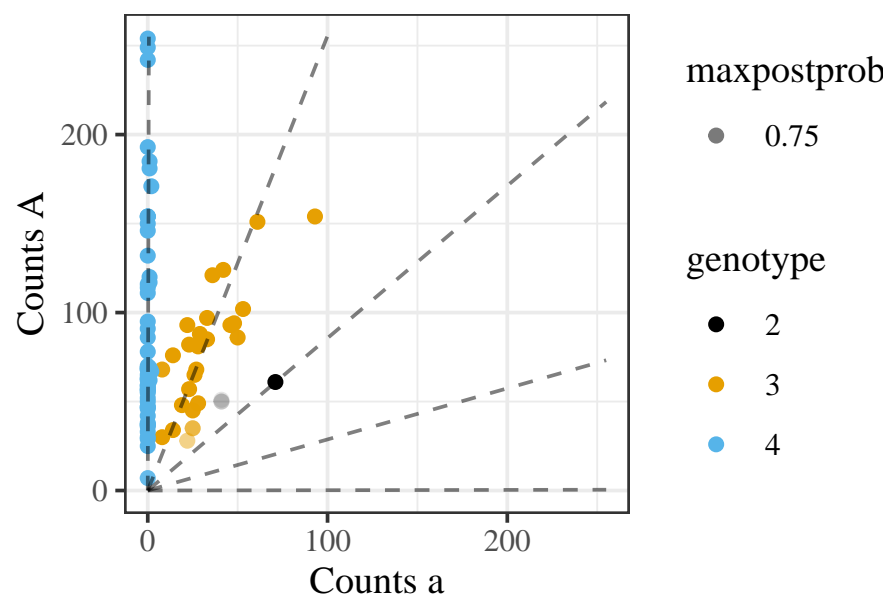

Figure S2: An annotated genotype plot, same as panel (B) of Figure 1 except a stronger penalty was applied to the bias parameter during the updog fit. This strengthened penalty resulted in more intuitive genotyping.

\begin{tabular}{|c|c|c|c|}
\hline Name & Probability Mass Function $f(k \mid \boldsymbol{\theta})$ & Parameterspace $\boldsymbol{\theta}$ & References \\
\hline $\begin{array}{l}\text { Discrete } \\
\text { Uniform }\end{array}$ & $1 /(K+1)$ & $\emptyset$ & McKenna et al. [2010] \\
\hline F1 & 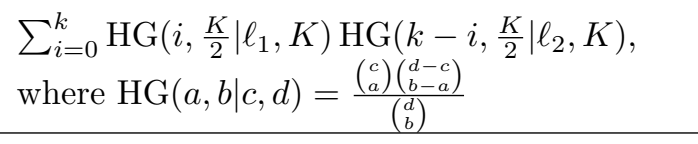 & $\ell_{1}, \ell_{2} \in\{0,1, \ldots, K\}$ & Serang et al. [2012] \\
\hline Binomial & $\left(\begin{array}{c}K \\
k\end{array}\right) \alpha^{k}(1-\alpha)^{K-k}$ & $\alpha \in[0,1]$ & $\begin{array}{l}\text { Martin et al. [2010], } \\
\text { Li [2011] }\end{array}$ \\
\hline Beta-binomial & $\left(\begin{array}{c}K \\
k\end{array}\right) \frac{\mathrm{B}\left(k+\alpha \frac{1-\gamma}{\gamma}, K-k+(1-\alpha) \frac{1-\gamma}{\gamma}\right)}{\mathrm{B}\left(\alpha \frac{1-\gamma}{\gamma},(1-\alpha) \frac{1-\gamma}{\gamma}\right)}$ & $\alpha, \gamma \in[0,1]$ & Blischak et al. [2018] \\
\hline $\begin{array}{l}\text { Proportional } \\
\text { Normal }\end{array}$ & $\frac{\mathrm{N}\left(k \mid \mu, \sigma^{2}\right)}{\sum_{k=0}^{K} \mathrm{~N}\left(k \mid \mu, \sigma^{2}\right)}$ & $\mu \in[0, K]$ and $\sigma^{2} \in \mathbb{R}^{+}$ & Current Paper \\
\hline Unimodal & $\begin{array}{l}\sum_{i=0}^{K} \pi_{i} \frac{1(k \in \mathcal{F}(a, i))}{|\mathcal{F}(a, i)|}, \text { where } \\
\mathcal{F}(a, b)=\{x \in \mathbb{Z}: x \in[\min (a, b), \max (a, b)]\}\end{array}$ & $\begin{array}{l}a \in(-1, K+1) \backslash\{0,1, \ldots, K\} \text { and } \\
\pi_{i} \in[0,1] \text { such that } \sum_{i=0}^{K} \pi_{i}=1\end{array}$ & Current Paper \\
\hline $\begin{array}{l}\text { General } \\
\text { Categorical }\end{array}$ & $\pi_{k}$ & $\pi_{k} \in[0,1]$ such that $\sum_{k} \pi_{k}=1$ & $\begin{array}{l}\text { DePristo et al. [2011] } \\
\mathrm{Li}[2011]\end{array}$ \\
\hline
\end{tabular}

Table S2: Prior distributions considered in this paper. We list their names, probability mass functions, the parameterspaces of the prior-specific parameters $\boldsymbol{\theta}$, and references where these priors were used for genotyping. 


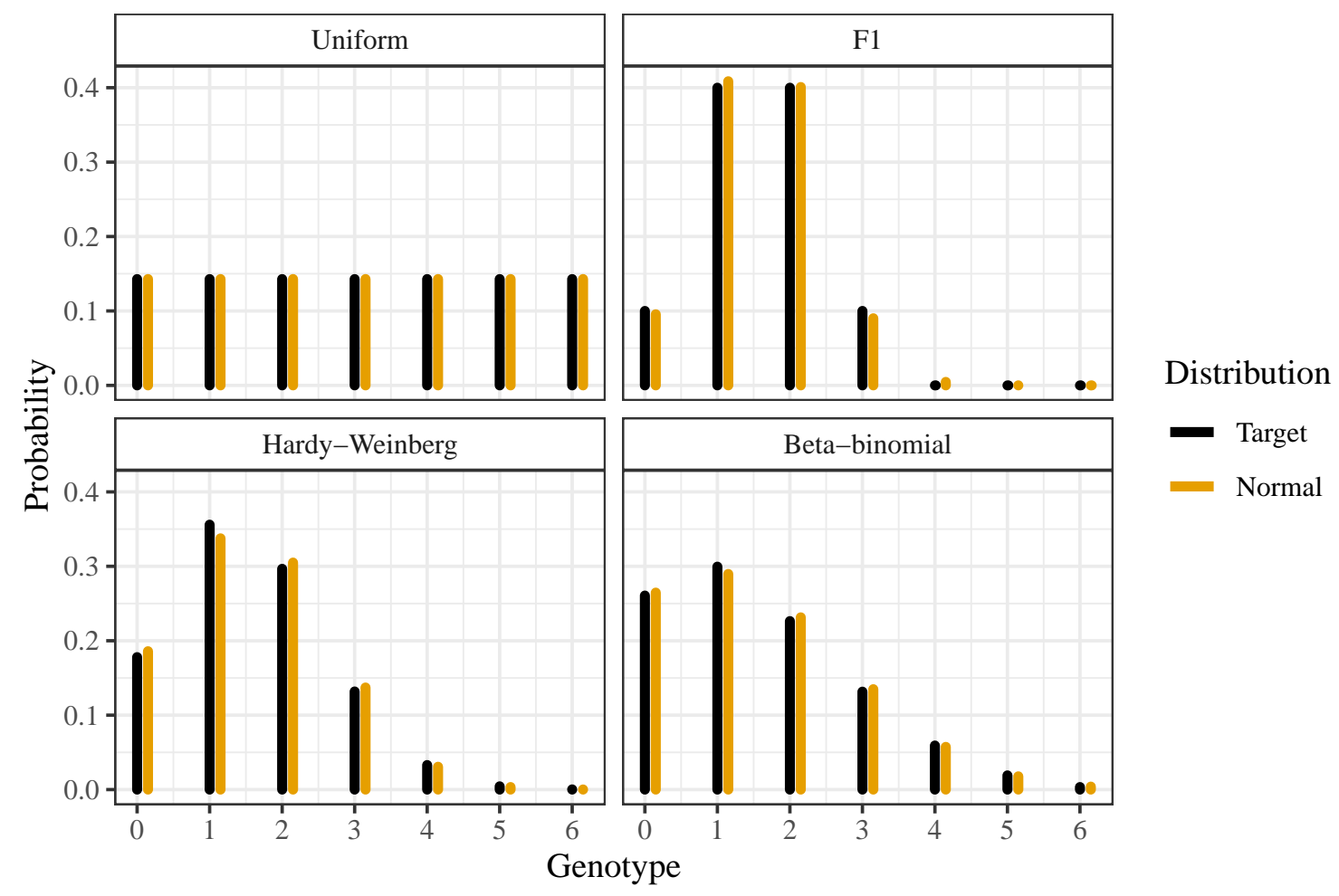

Figure S3: Four distributions of genotypes (black) and the closest distribution in the class of proportional normal distributions (orange). Closeness was measured by Kullback-Leibler divergence. Elements in the class of proportional normal distributions approximate very well the discrete uniform, the F1 $\left(\ell_{1}=1\right.$ and $\left.\ell_{2}=2\right)$, the binomial $(\alpha=0.25)$, and the beta-binomial $(\alpha=0.25$ and $\gamma=0.01)$ distributions. The parameter values of the plotted proportional normal distributions are given in Supplementary Table S1. 

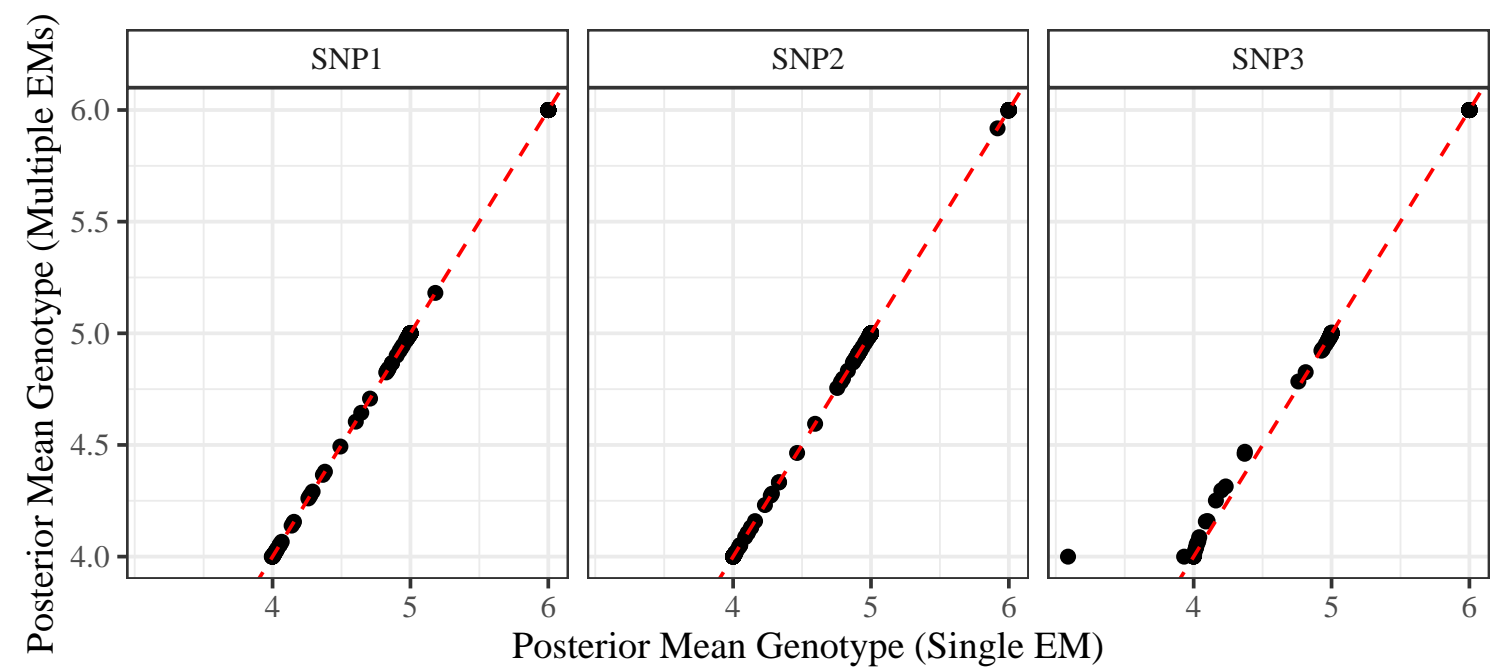

Figure S4: Posterior mean genotypes for the three example SNPs used in Gerard et al. [2018]. These were calculated using either the single EM approach with a small discrete uniform mixing component ( $x$-axis) or the multiple EM approach that iterates over all possible parental genotypes ( $y$-axis). The dashed red line is the $y=x$ line. The posterior mean genotypes from the two approaches are nearly identical.
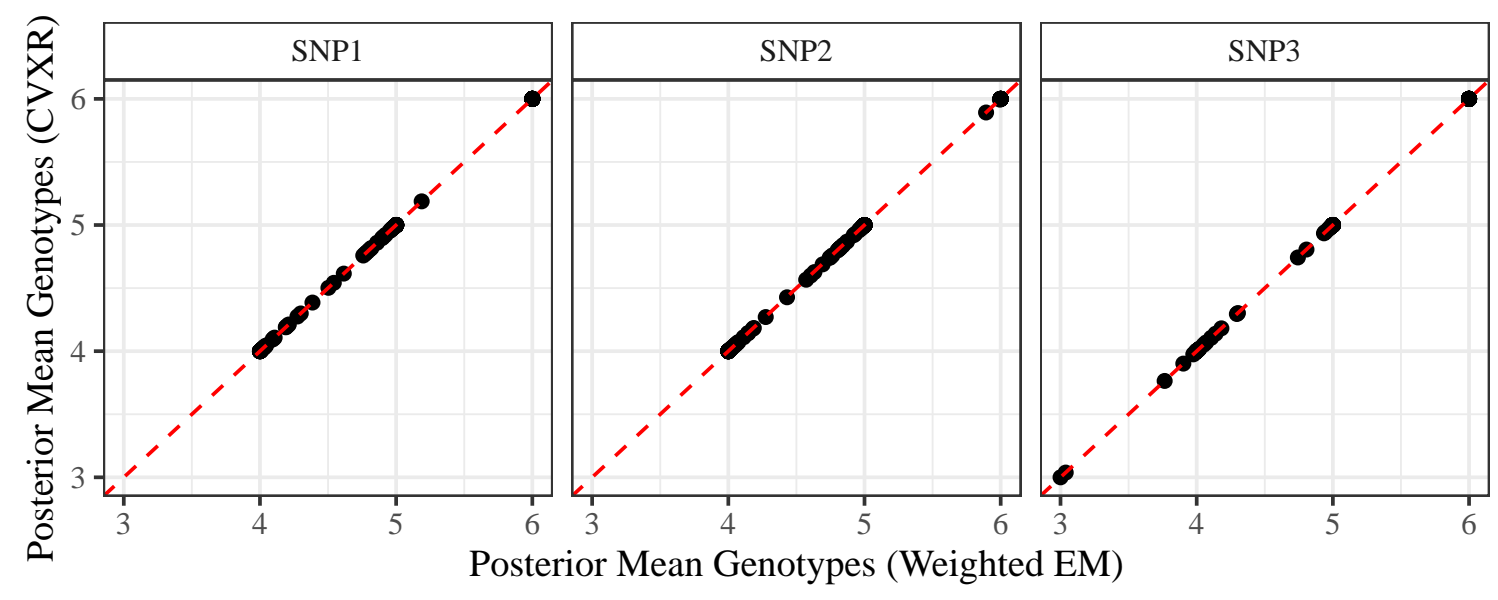

Figure S5: Posterior mean genotypes for the three example SNPs used in Gerard et al. [2018]. These were calculated using either the weighted EM approach for the class of unimodal distributions $(x$ axis) or using interior point methods ( $y$-axis). The dashed red line is the $y=x$ line. The posterior mean genotypes from the two approaches are nearly identical. 

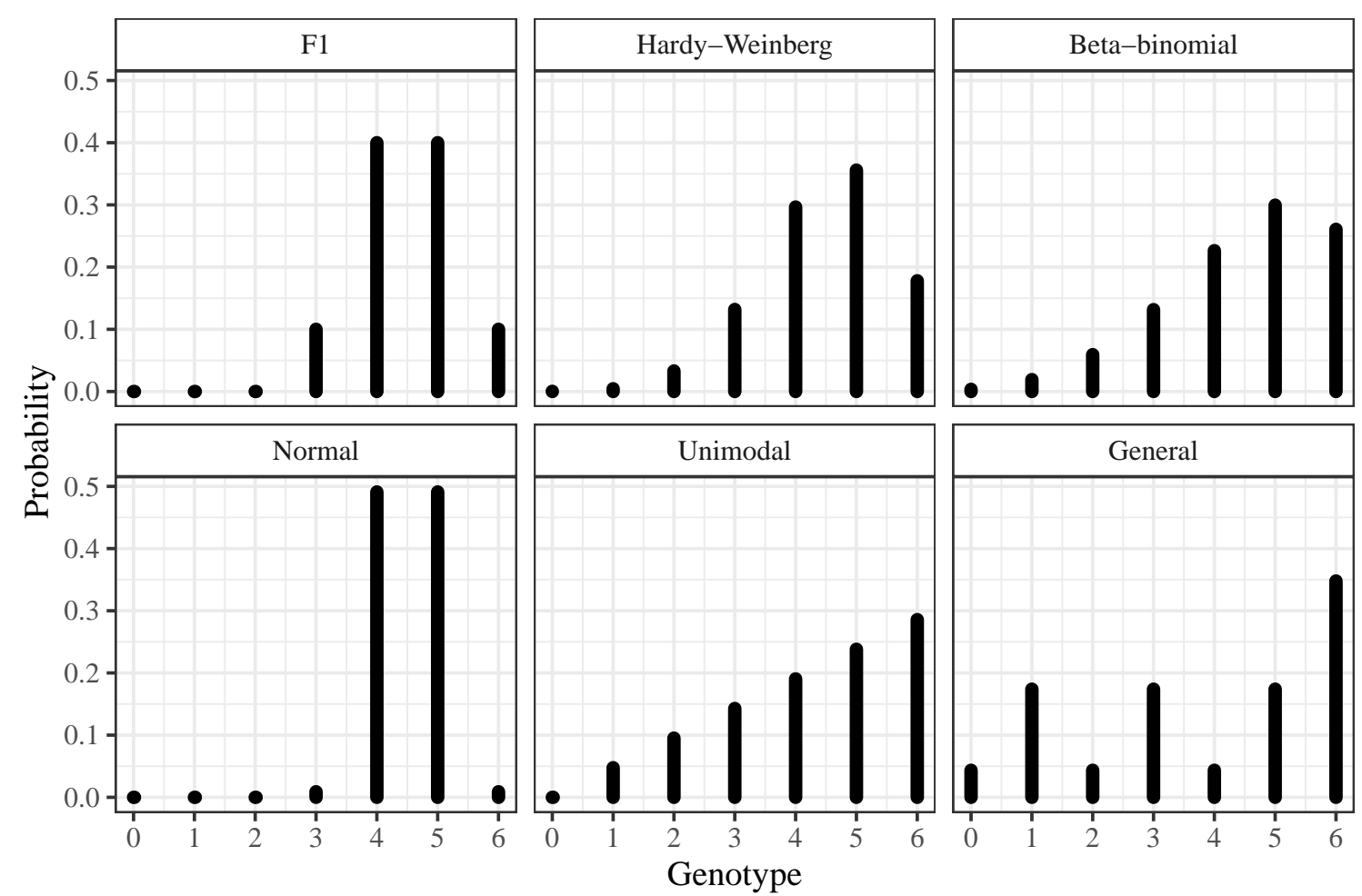

Figure S6: True genotype distributions used in the simulation study of Section 3, explicitly defined in Supplementary Section S3. Each genotype distribution was chosen to be favorable to one of the assumed classes of priors: the class of F1 distributions, the class of binomial (Hardy-Weinberg) distributions, the class of beta-binomial distributions, the class of proportional normal distributions, the class of unimodal distributions, and the class of general distributions. Not plotted is the discrete uniform distribution, also used in the simulation study. 
bioRxiv preprint doi: https://doi.org/10.1101/751784; this version posted August 31, 2019. The copyright holder for this preprint (which was not
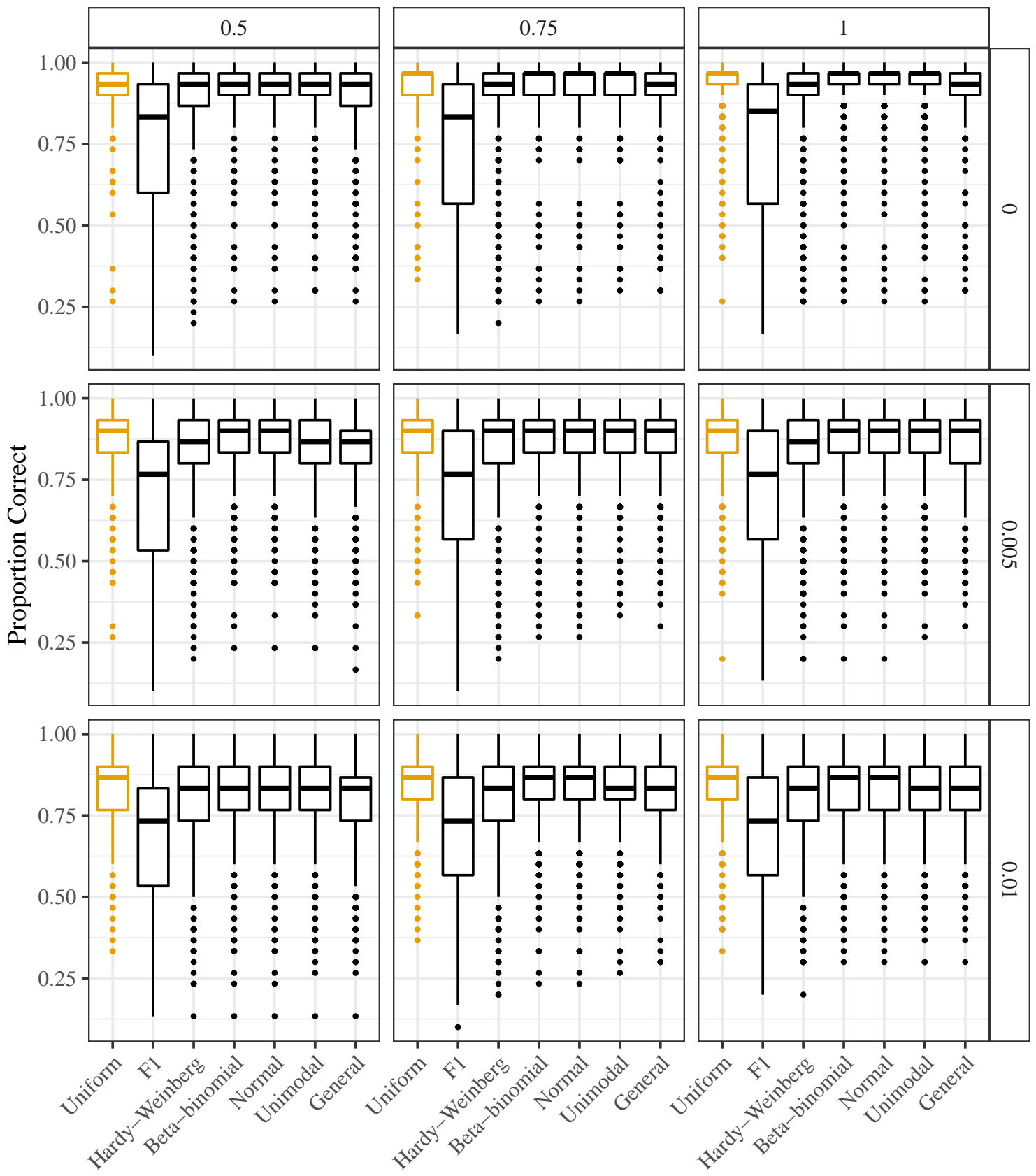

Assumed Prior

Figure S7: Proportion of individuals genotyped correctly ( $y$-axis) stratified by the assumed class of prior distribution ( $x$-axis). Column facets index varying levels of allele bias (with 1 indicating no bias) and row facets index varying levels of overdispersion (with 0 indicating no overdispersion). The genotypes were generated from a distribution designed to be favorable to the discrete uniform prior distribution (orange). 
bioRxiv preprint doi: https://doi.org/10.1101/751784; this version posted August 31, 2019. The copyright holder for this preprint (which was not
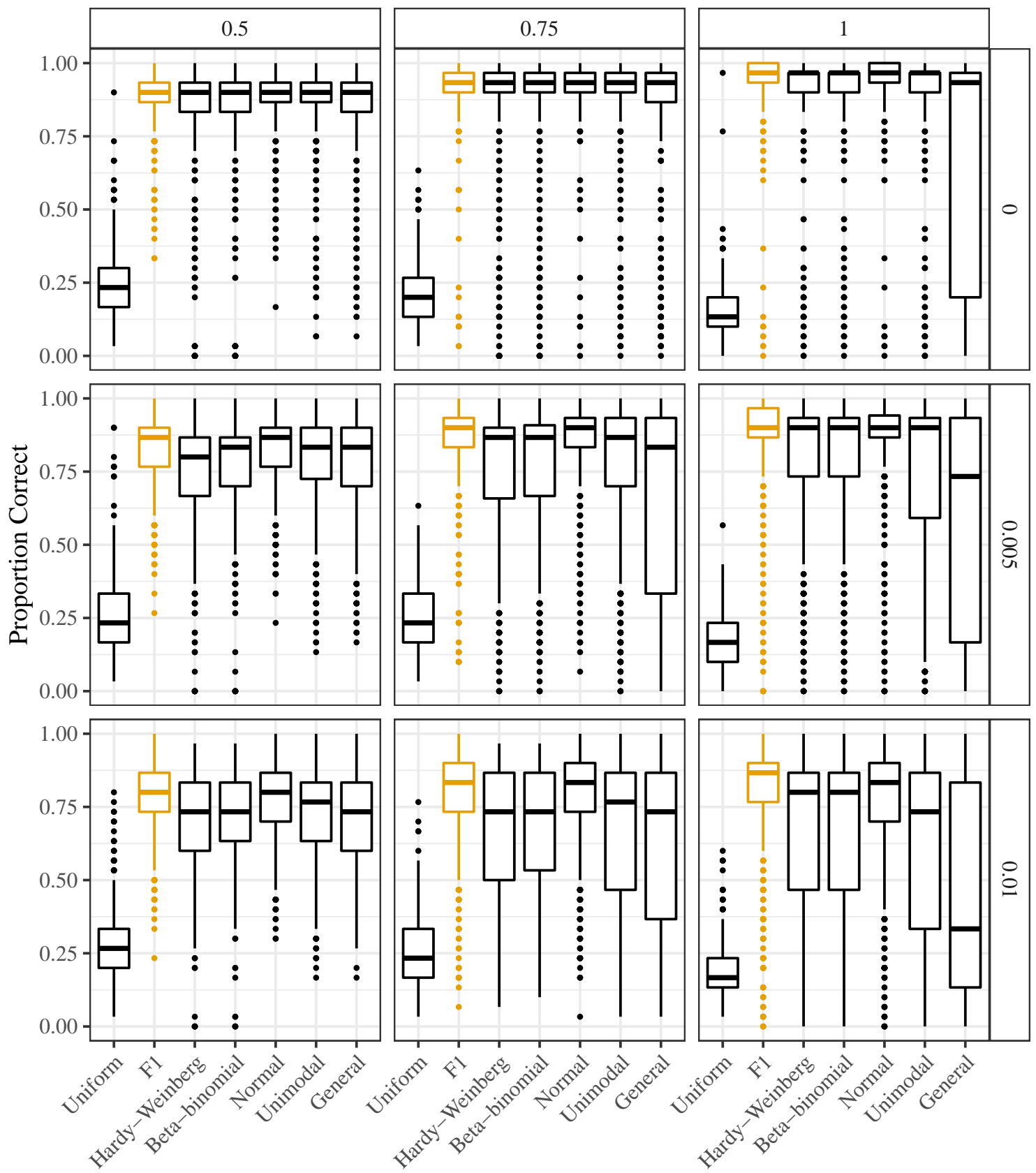

Assumed Prior

Figure S8: Proportion of individuals genotyped correctly ( $y$-axis) stratified by the assumed class of prior distribution ( $x$-axis). Column facets index varying levels of allele bias (with 1 indicating no bias) and row facets index varying levels of overdispersion (with 0 indicating no overdispersion). The genotypes were generated from a distribution designed to be favorable to the F1 class of prior distributions (orange). 
bioRxiv preprint doi: https://doi.org/10.1101/751784; this version posted August 31, 2019. The copyright holder for this preprint (which was not
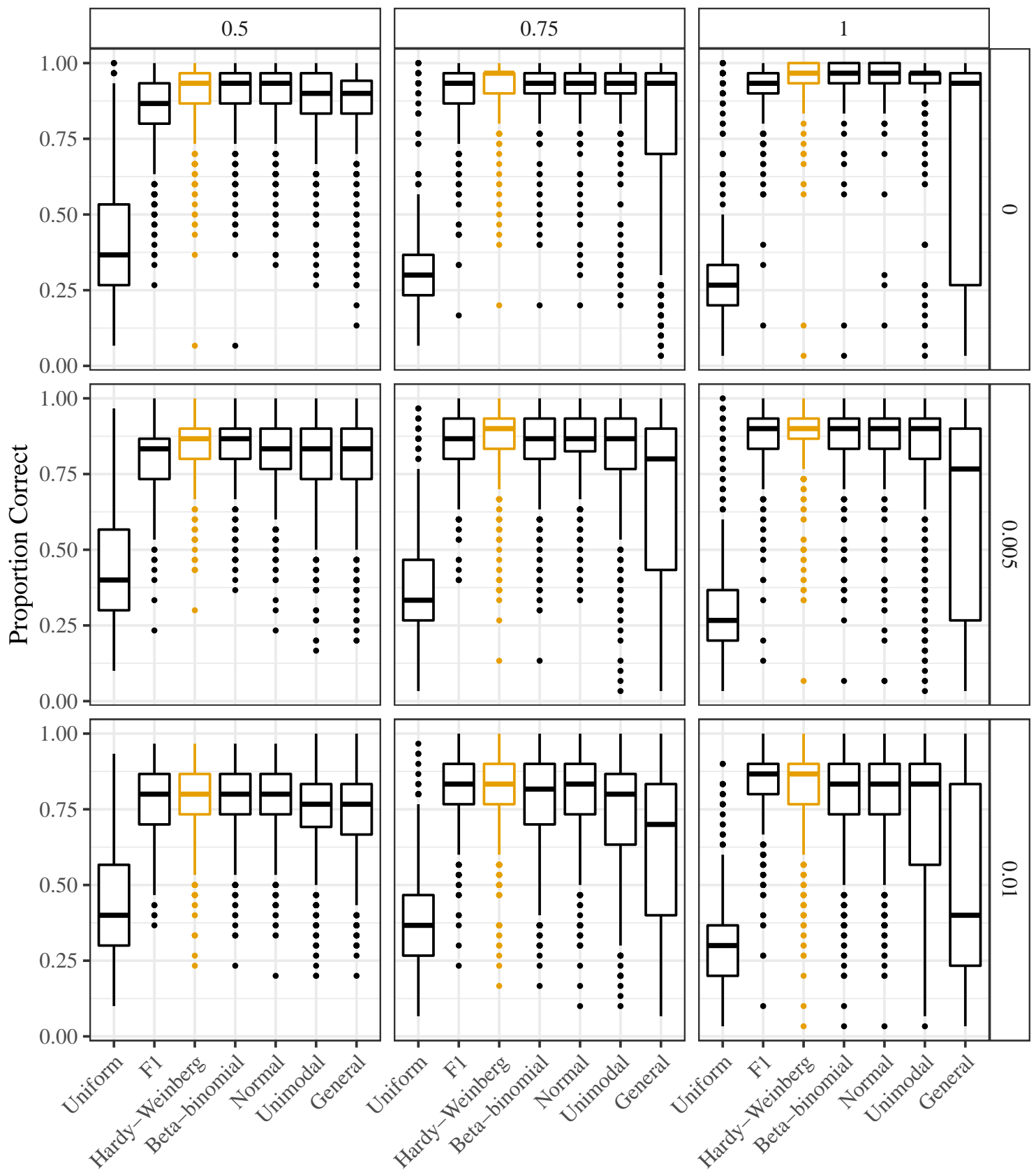

Assumed Prior

Figure S9: Proportion of individuals genotyped correctly ( $y$-axis) stratified by the assumed class of prior distribution $(x$-axis). Column facets index varying levels of allele bias (with 1 indicating no bias) and row facets index varying levels of overdispersion (with 0 indicating no overdispersion). The genotypes were generated from a distribution designed to be favorable to the binomial (HardyWeinberg) class of prior distributions (orange). 
bioRxiv preprint doi: https://doi.org/10.1101/751784; this version posted August 31, 2019. The copyright holder for this preprint (which was not
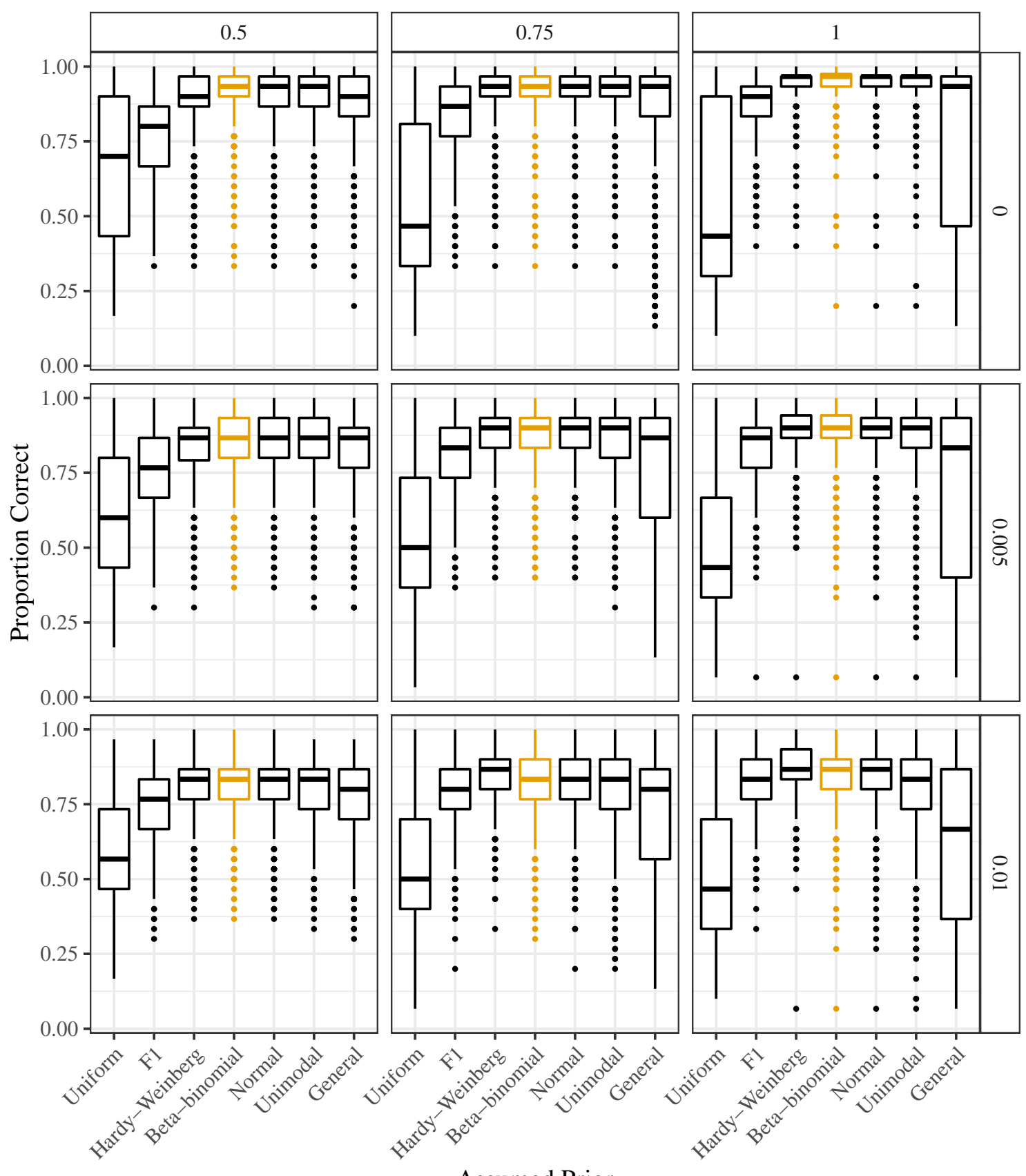

Assumed Prior

Figure S10: Proportion of individuals genotyped correctly ( $y$-axis) stratified by the assumed class of prior distribution ( $x$-axis). Column facets index varying levels of allele bias (with 1 indicating no bias) and row facets index varying levels of overdispersion (with 0 indicating no overdispersion). The genotypes were generated from a distribution designed to be favorable to the beta-binomial class of prior distributions (orange). 

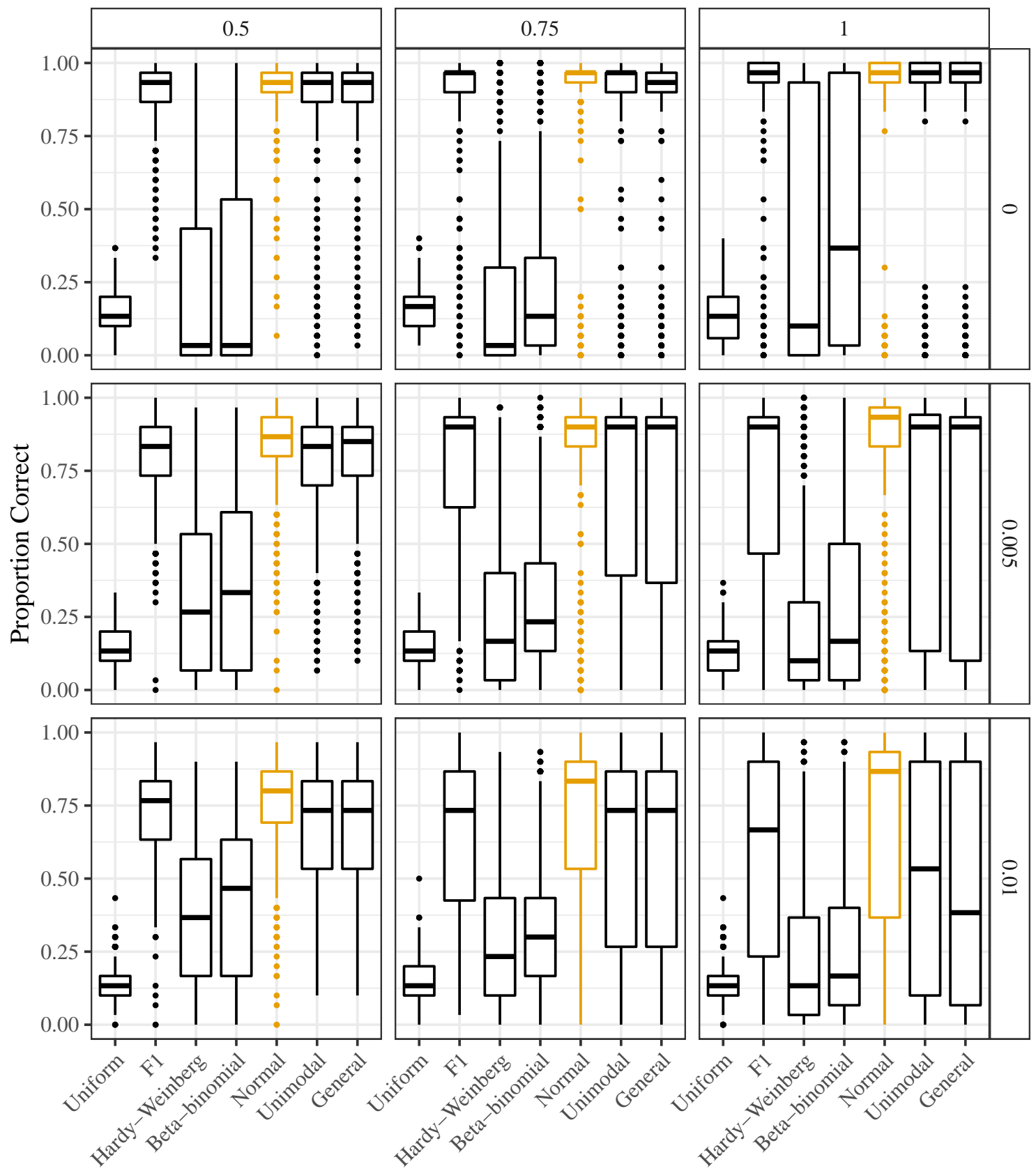

Assumed Prior

Figure S11: Proportion of individuals genotyped correctly ( $y$-axis) stratified by the assumed class of prior distribution ( $x$-axis). Column facets index varying levels of allele bias (with 1 indicating no bias) and row facets index varying levels of overdispersion (with 0 indicating no overdispersion). The genotypes were generated from a distribution designed to be favorable to the proportional normal class of prior distributions (orange). 
bioRxiv preprint doi: https://doi.org/10.1101/751784; this version posted August 31, 2019. The copyright holder for this preprint (which was not
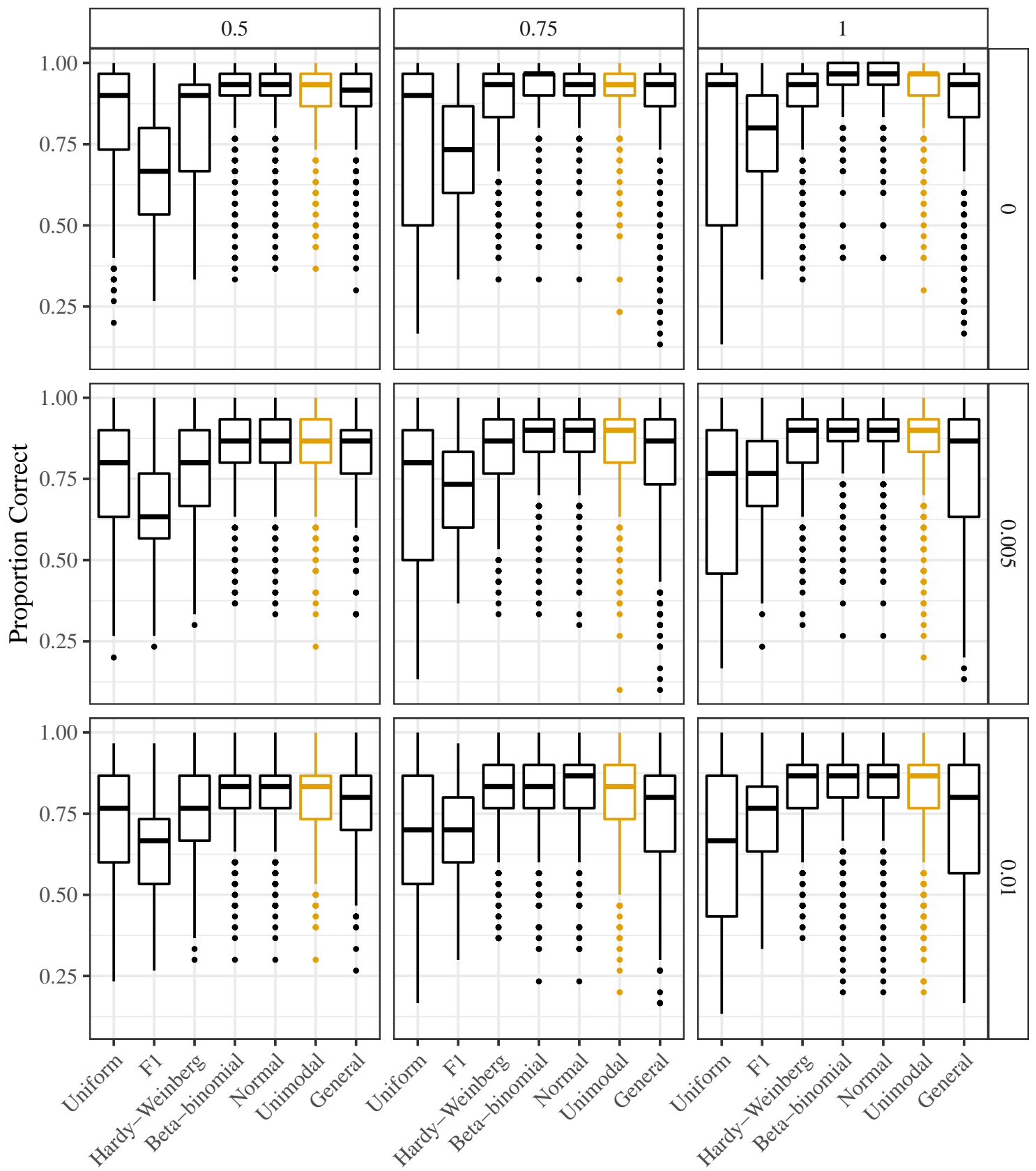

\section{Assumed Prior}

Figure S12: Proportion of individuals genotyped correctly ( $y$-axis) stratified by the assumed class of prior distribution ( $x$-axis). Column facets index varying levels of allele bias (with 1 indicating no bias) and row facets index varying levels of overdispersion (with 0 indicating no overdispersion). The genotypes were generated from a distribution designed to be favorable to the unimodal class of prior distributions (orange). 
bioRxiv preprint doi: https://doi.org/10.1101/751784; this version posted August 31, 2019. The copyright holder for this preprint (which was not
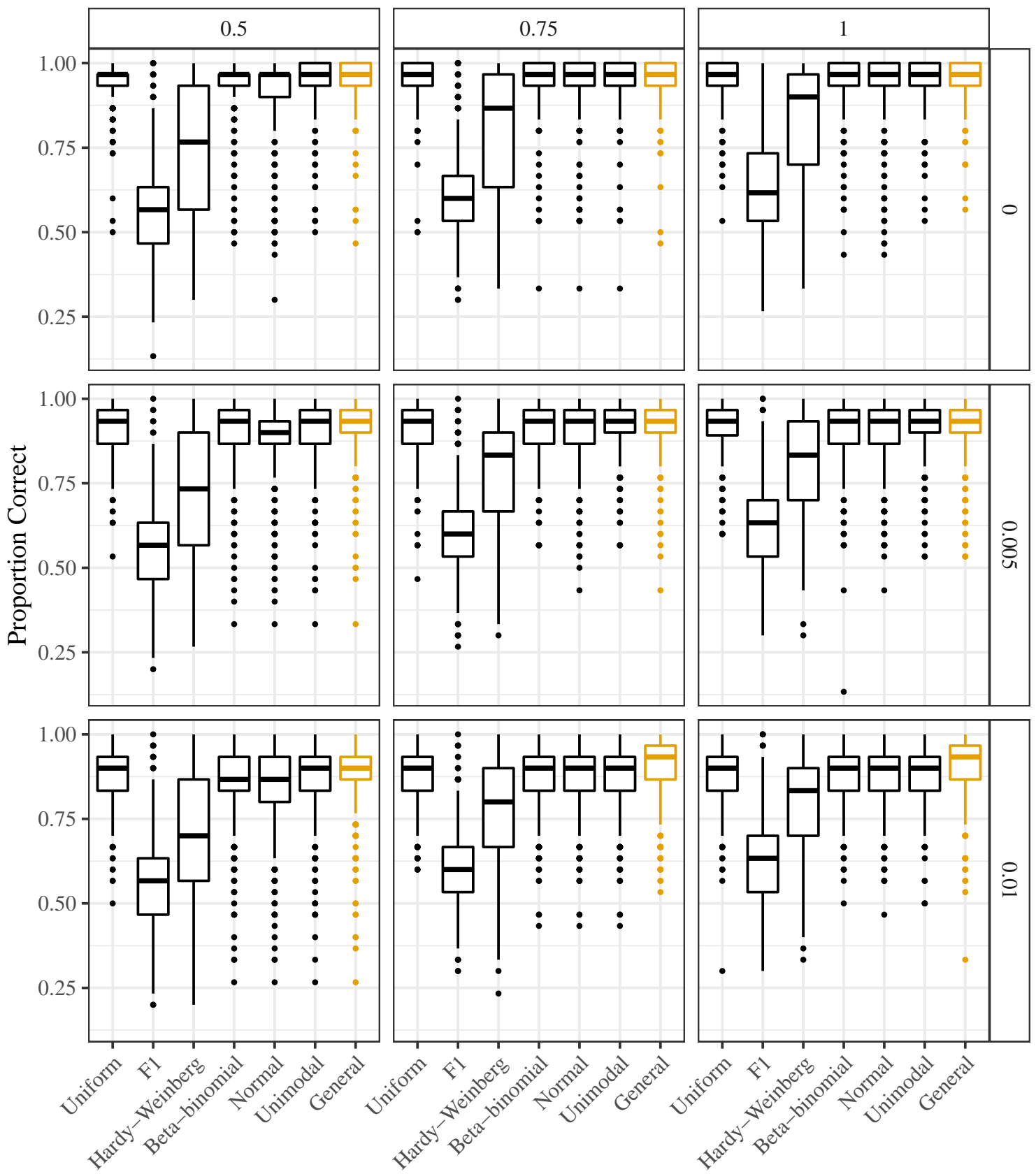

Assumed Prior

Figure S13: Proportion of individuals genotyped correctly ( $y$-axis) stratified by the assumed class of prior distributions ( $x$-axis). Column facets index varying levels of allele bias (with 1 indicating no bias) and row facets index varying levels of overdispersion (with 0 indicating no overdispersion). The genotypes were generated from a distribution designed to be favorable to the general class of prior distributions (orange). 
bioRxiv preprint doi: https://doi.org/10.1101/751784; this version posted August 31, 2019. The copyright holder for this preprint (which was not
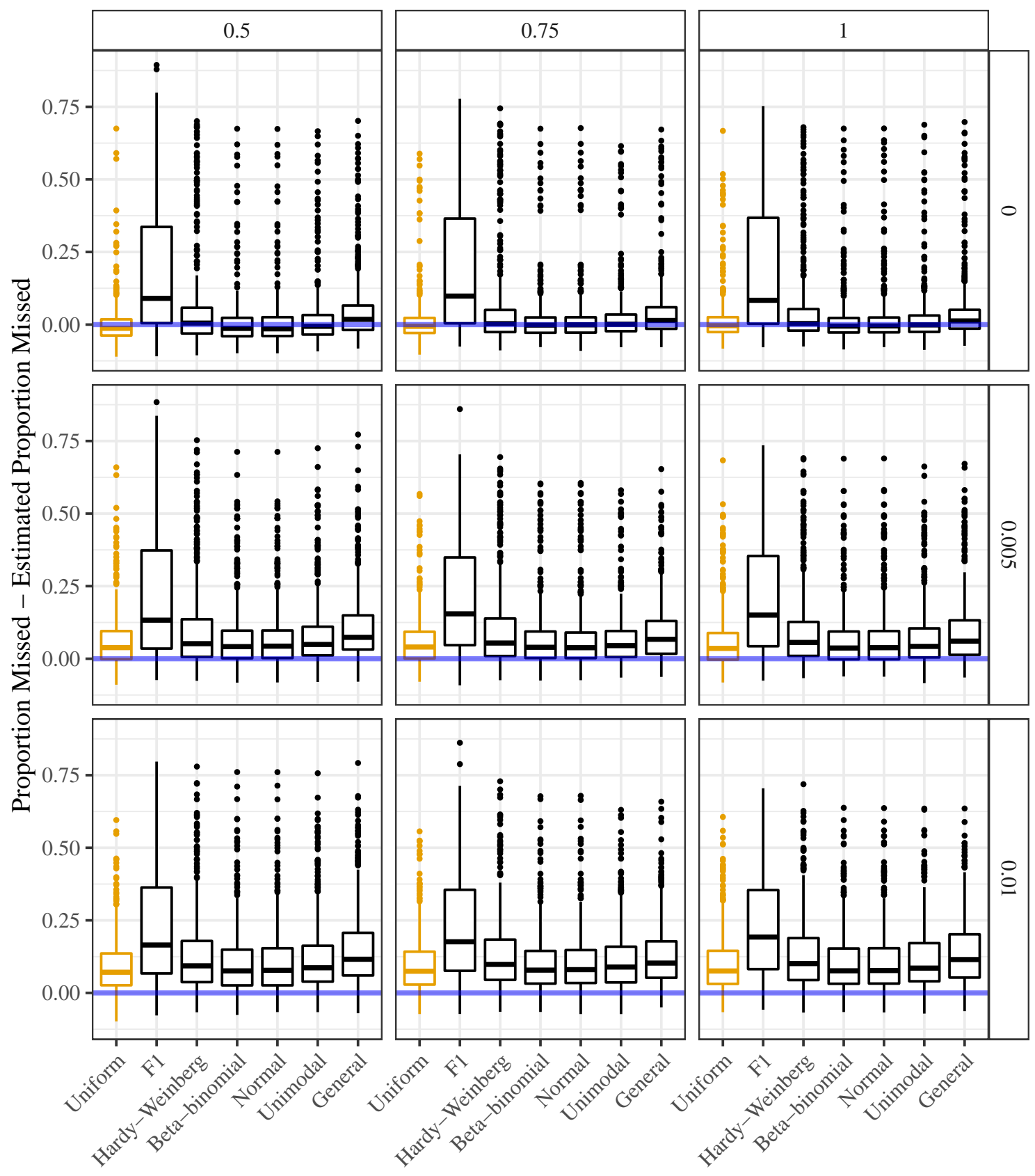

Assumed Prior

Figure S14: Estimated proportion of individuals incorrectly genotyped subtracted from the actual proportion of individuals incorrectly genotyped ( $y$-axis) stratified by the assumed class of prior distributions $(x$-axis). The horizontal line (at $y=0)$ indicates unbiased estimation of the misclassification error rate. Column facets index varying levels of allele bias (with 1 indicating no bias) and row facets index varying levels of overdispersion (with 0 indicating no overdispersion). The genotypes were generated from a distribution designed to be favorable to the discrete uniform prior distribution (orange). 

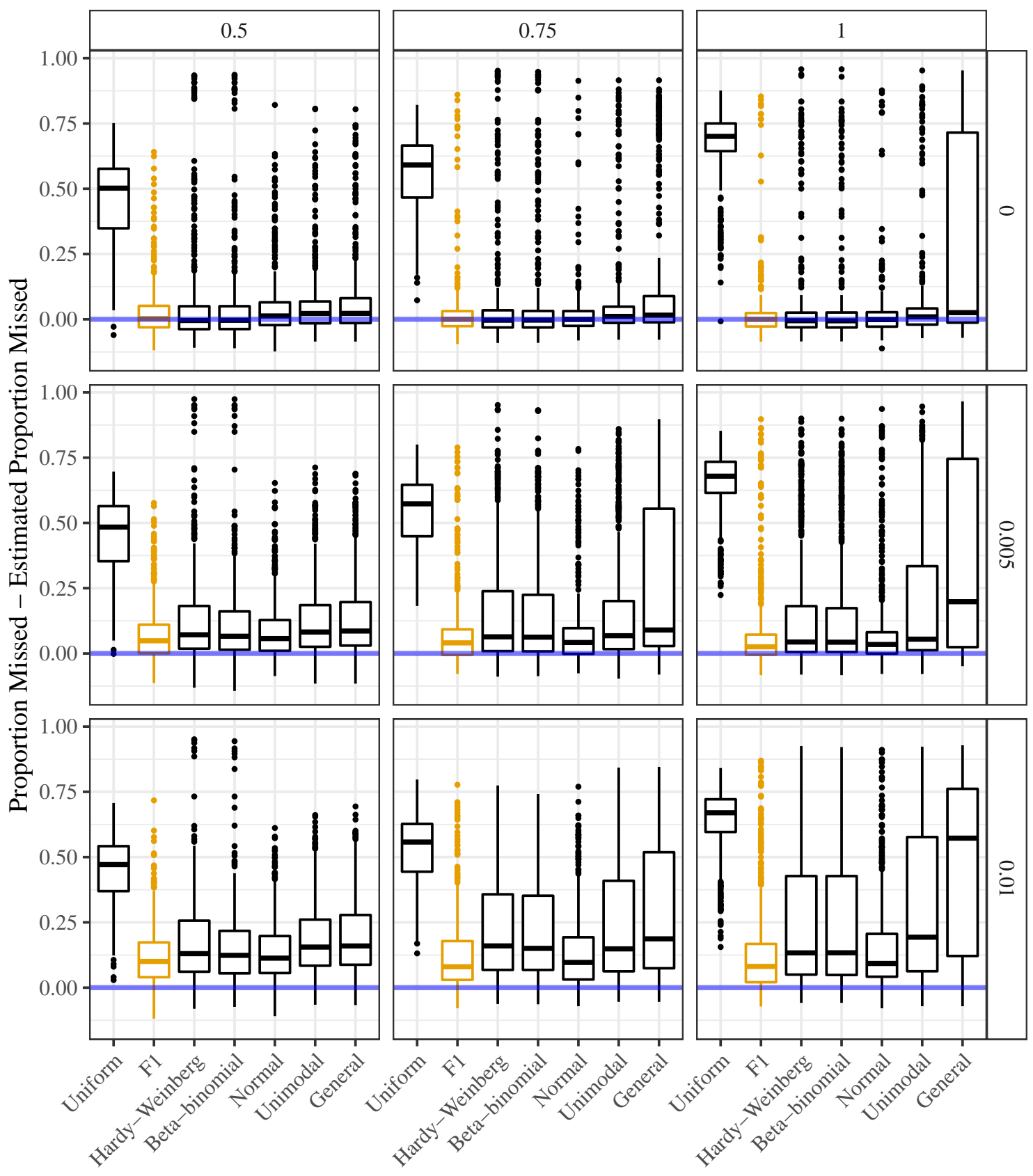

Assumed Prior

Figure S15: Estimated proportion of individuals incorrectly genotyped subtracted from the actual proportion of individuals incorrectly genotyped ( $y$-axis) stratified by the assumed class of prior distributions ( $x$-axis). The horizontal line (at $y=0$ ) indicates unbiased estimation of the misclassification error rate. Column facets index varying levels of allele bias (with 1 indicating no bias) and row facets index varying levels of overdispersion (with 0 indicating no overdispersion). The genotypes were generated from a distribution designed to be favorable to the class of F1 prior distributions (orange). 

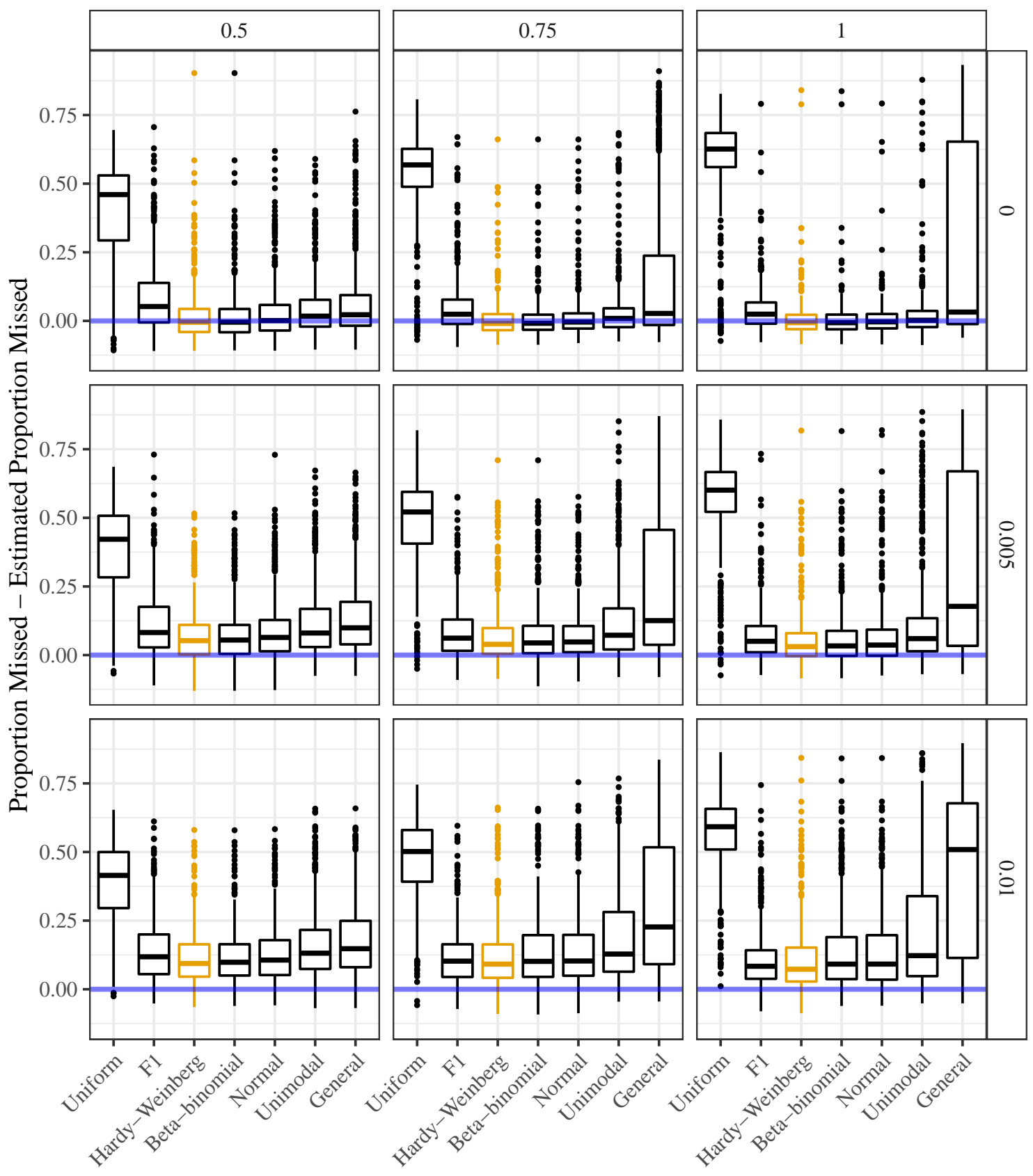

Assumed Prior

Figure S16: Estimated proportion of individuals incorrectly genotyped subtracted from the actual proportion of individuals incorrectly genotyped ( $y$-axis) stratified by the assumed class of prior distributions $(x$-axis). The horizontal line (at $y=0)$ indicates unbiased estimation of the misclassification error rate. Column facets index varying levels of allele bias (with 1 indicating no bias) and row facets index varying levels of overdispersion (with 0 indicating no overdispersion). The genotypes were generated from a distribution designed to be favorable to the class of binomial (Hardy-Weinberg) prior distributions (orange). 

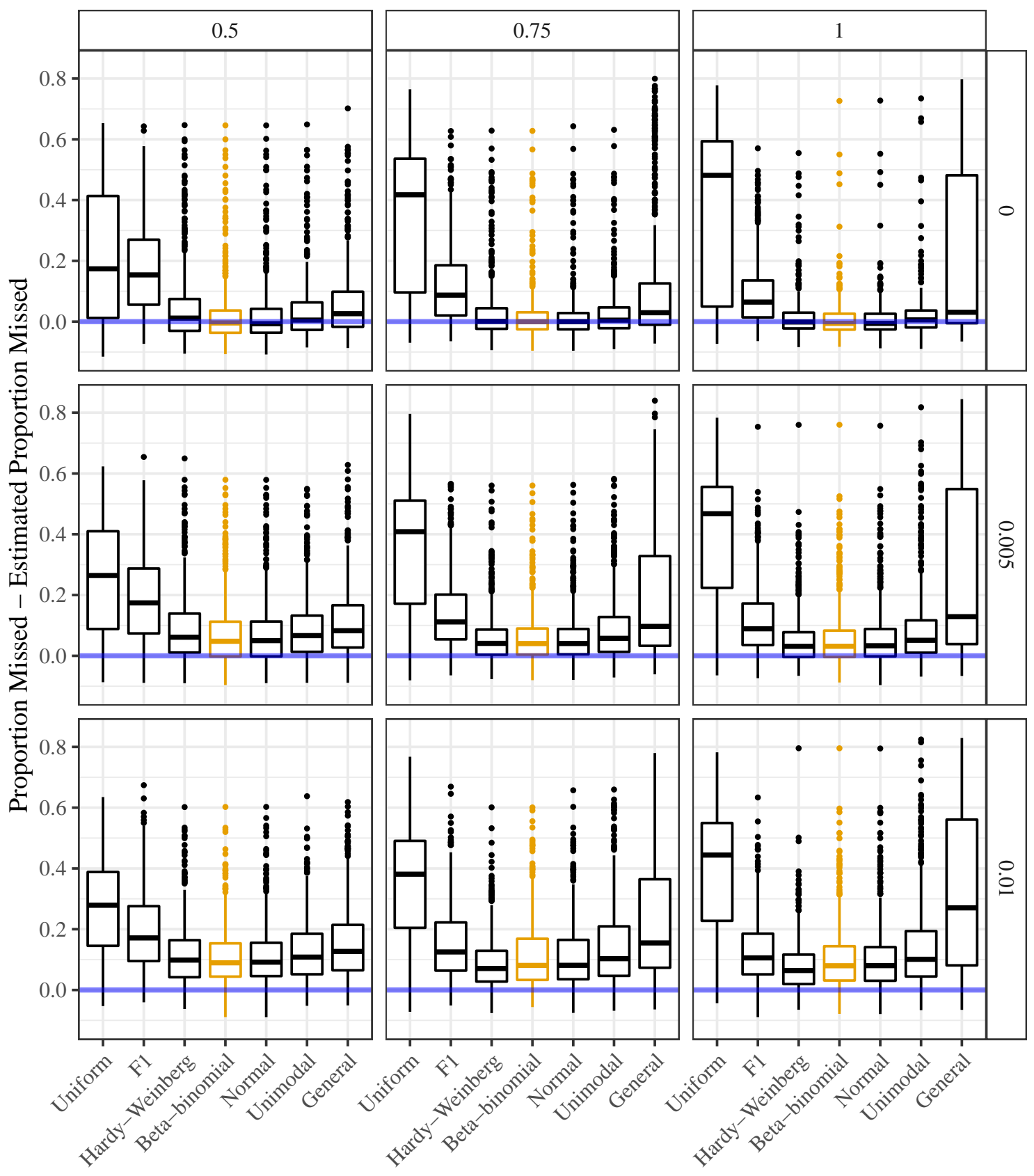

Assumed Prior

Figure S17: Estimated proportion of individuals incorrectly genotyped subtracted from the actual proportion of individuals incorrectly genotyped ( $y$-axis) stratified by the assumed class of prior distributions $(x$-axis). The horizontal line (at $y=0)$ indicates unbiased estimation of the misclassification error rate. Column facets index varying levels of allele bias (with 1 indicating no bias) and row facets index varying levels of overdispersion (with 0 indicating no overdispersion). The genotypes were generated from a distribution designed to be favorable to the class of beta-binomial prior distributions (orange). 

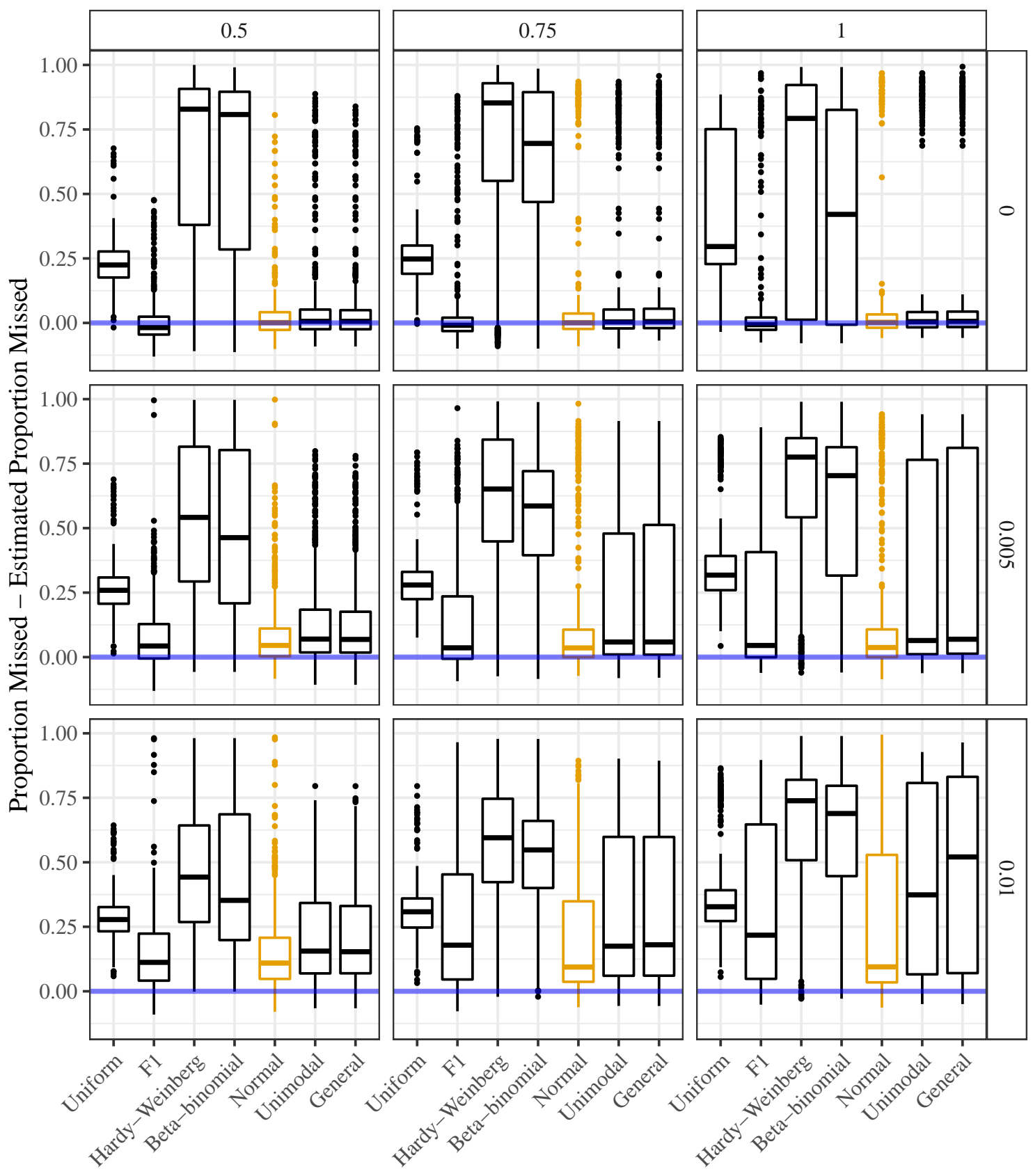

Assumed Prior

Figure S18: Estimated proportion of individuals incorrectly genotyped subtracted from the actual proportion of individuals incorrectly genotyped ( $y$-axis) stratified by the assumed class of prior distributions $(x$-axis). The horizontal line (at $y=0)$ indicates unbiased estimation of the misclassification error rate. Column facets index varying levels of allele bias (with 1 indicating no bias) and row facets index varying levels of overdispersion (with 0 indicating no overdispersion). The genotypes were generated from a distribution designed to be favorable to the class of proportional normal prior distributions (orange). 
bioRxiv preprint doi: https://doi.org/10.1101/751784; this version posted August 31, 2019. The copyright holder for this preprint (which was not
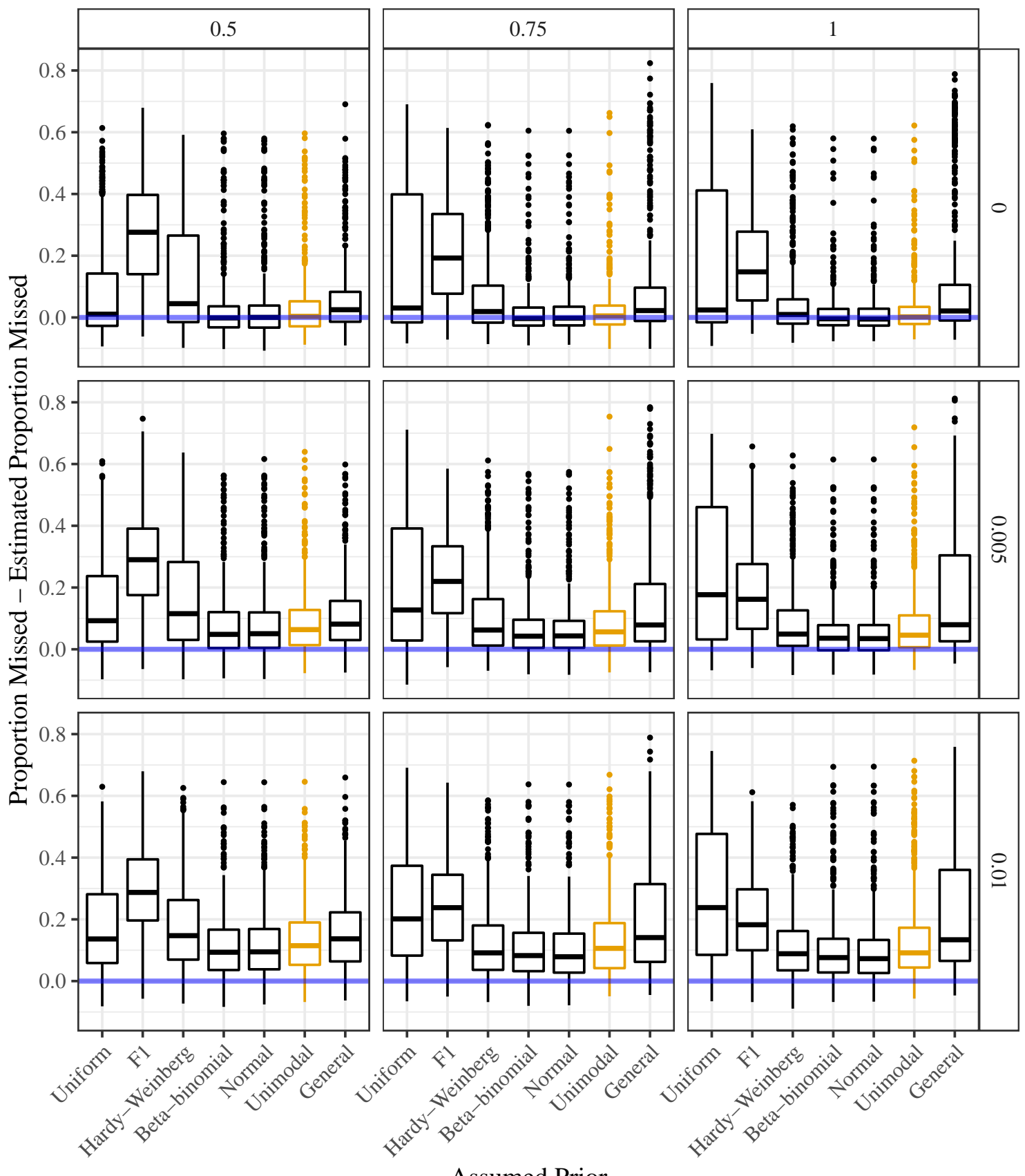

Assumed Prior

Figure S19: Estimated proportion of individuals incorrectly genotyped subtracted from the actual proportion of individuals incorrectly genotyped ( $y$-axis) stratified by the assumed class of prior distributions $(x$-axis). The horizontal line (at $y=0)$ indicates unbiased estimation of the misclassification error rate. Column facets index varying levels of allele bias (with 1 indicating no bias) and row facets index varying levels of overdispersion (with 0 indicating no overdispersion). The genotypes were generated from a distribution designed to be favorable to the class of unimodal prior distributions (orange). 
bioRxiv preprint doi: https://doi.org/10.1101/751784; this version posted August 31, 2019. The copyright holder for this preprint (which was not
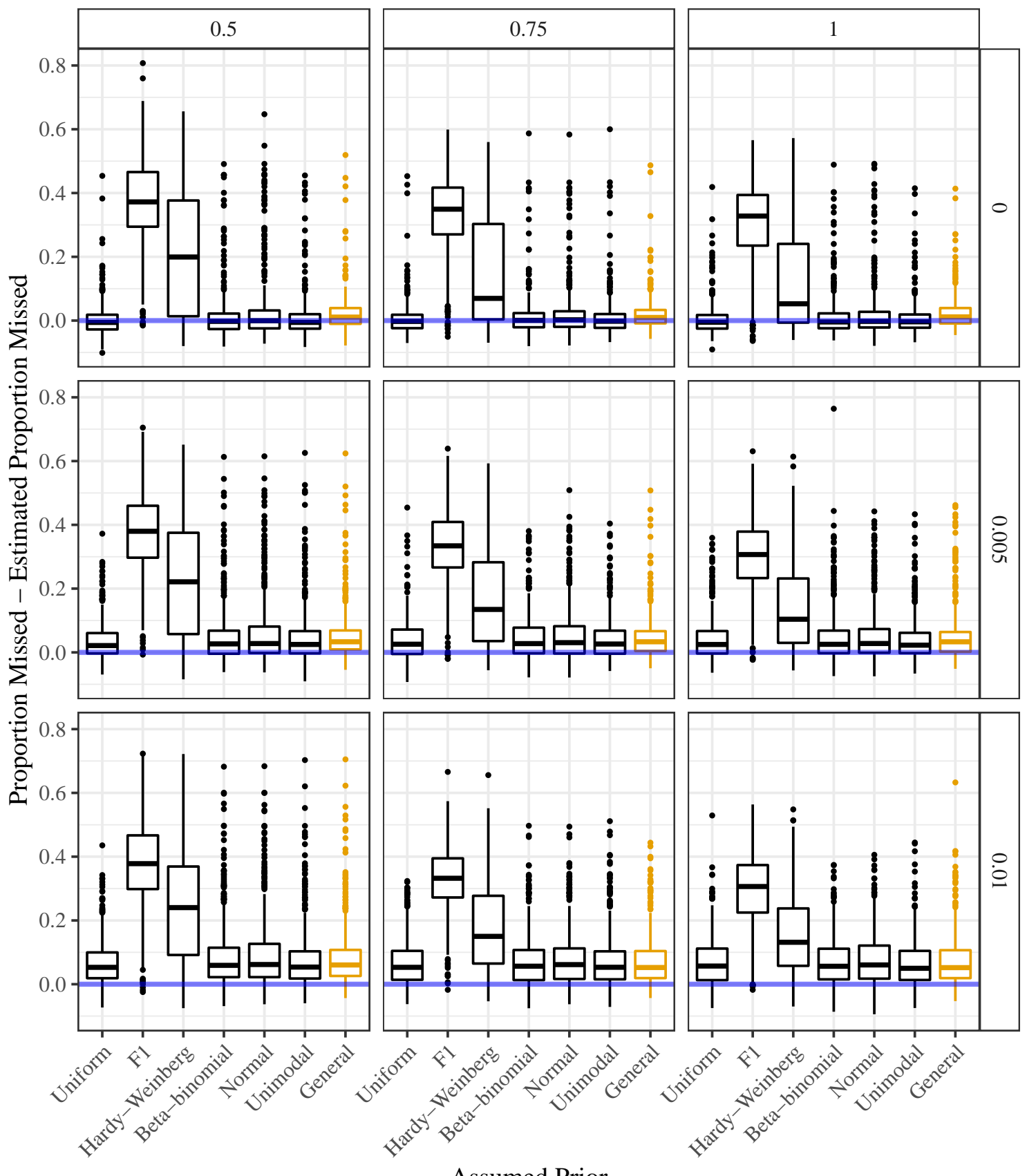

Assumed Prior

Figure S20: Estimated proportion of individuals incorrectly genotyped subtracted from the actual proportion of individuals incorrectly genotyped ( $y$-axis) stratified by the assumed class of prior distributions ( $x$-axis). The horizontal line (at $y=0)$ indicates unbiased estimation of the misclassification error rate. Column facets index varying levels of allele bias (with 1 indicating no bias) and row facets index varying levels of overdispersion (with 0 indicating no overdispersion). The genotypes were generated from a distribution designed to be favorable to the class of general prior distributions (orange). 

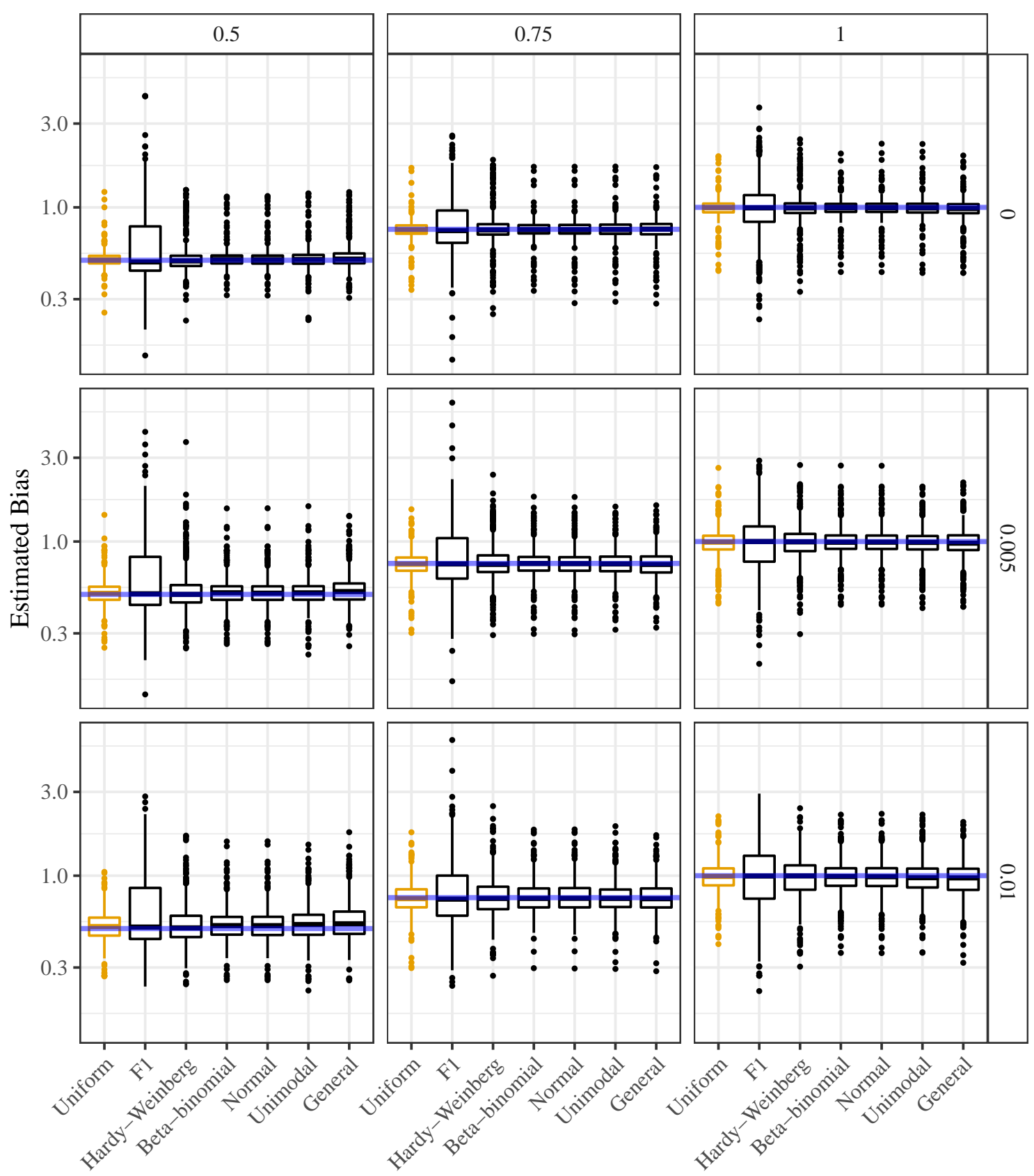

Assumed Prior

Figure S21: Estimated allele bias ( $y$-axis) stratified by the assumed class of prior distributions $(x-$ axis). The horizontal line is the actual allele bias. Column facets index varying levels of allele bias (with 1 indicating no bias) and row facets index varying levels of overdispersion (with 0 indicating no overdispersion). The genotypes were generated from a distribution designed to be favorable to the discrete uniform prior distribution (orange). 

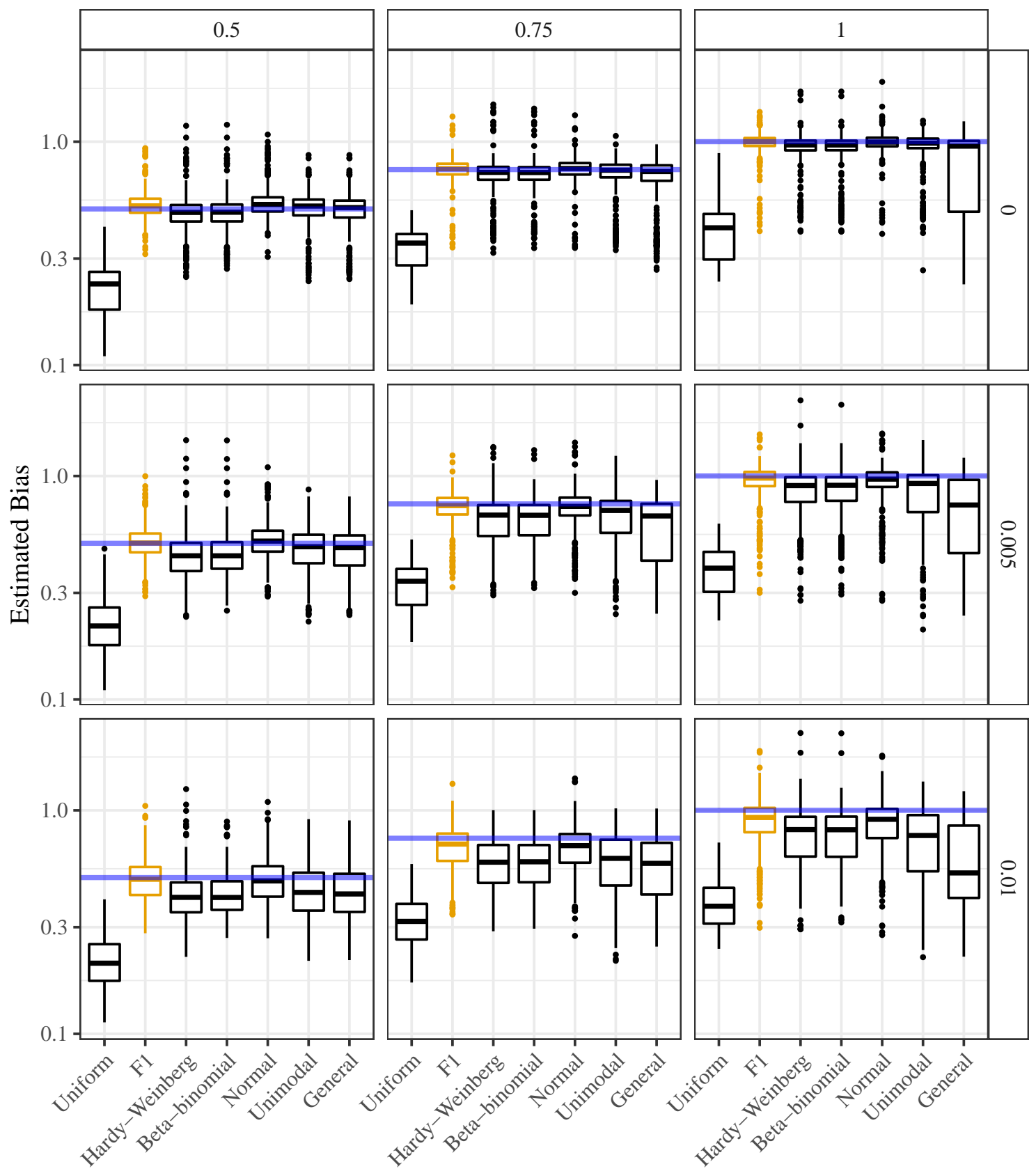

Assumed Prior

Figure S22: Estimated allele bias ( $y$-axis) stratified by the assumed class of prior distributions $(x-$ axis). The horizontal line is the actual allele bias. Column facets index varying levels of allele bias (with 1 indicating no bias) and row facets index varying levels of overdispersion (with 0 indicating no overdispersion). The genotypes were generated from a distribution designed to be favorable to the class of F1 prior distributions (orange). 

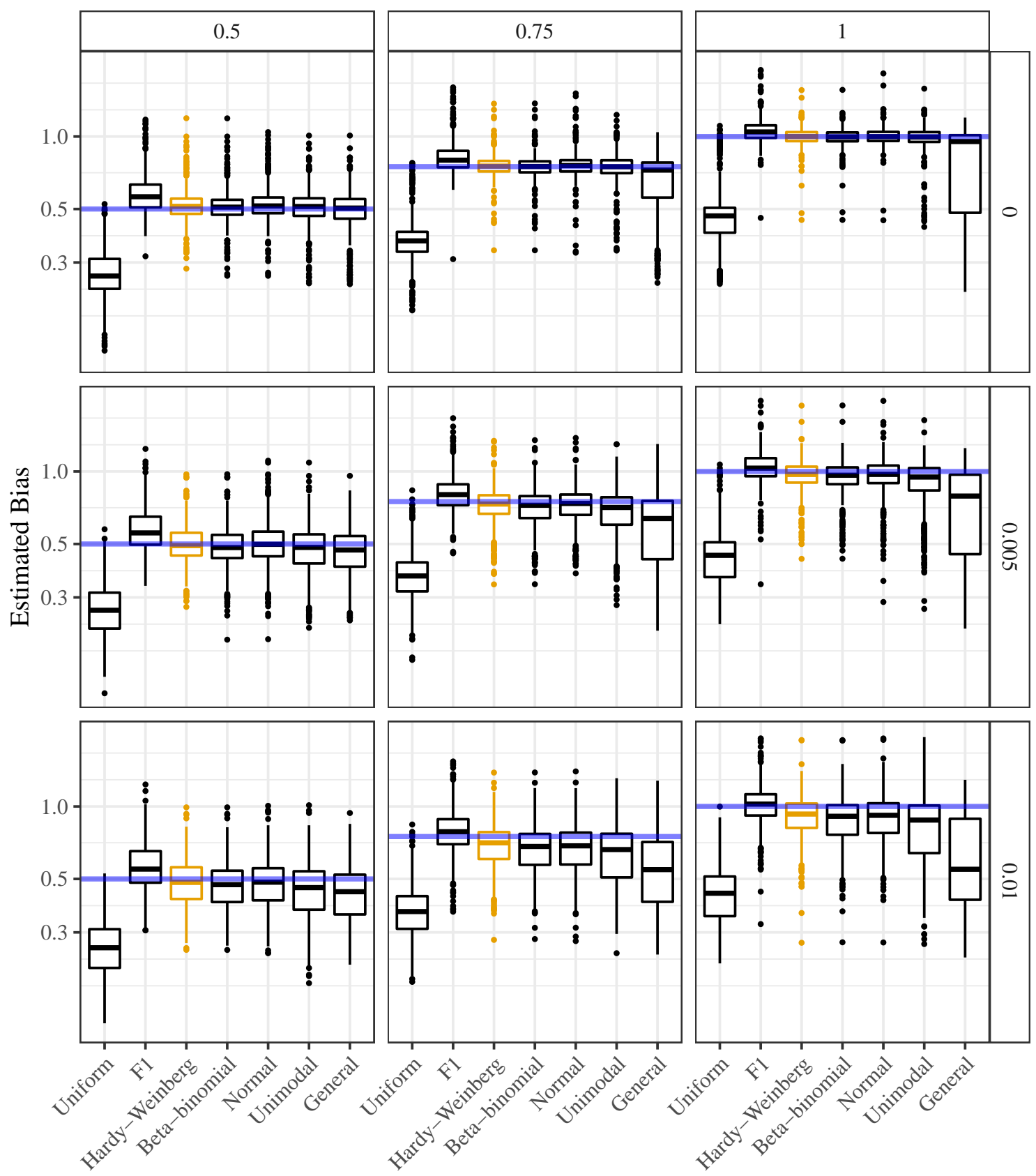

Assumed Prior

Figure S23: Estimated allele bias ( $y$-axis) stratified by the assumed class of prior distributions $(x-$ axis). The horizontal line is the actual allele bias. Column facets index varying levels of allele bias (with 1 indicating no bias) and row facets index varying levels of overdispersion (with 0 indicating no overdispersion). The genotypes were generated from a distribution designed to be favorable to the class of binomial (Hardy-Weinberg) prior distributions (orange). 

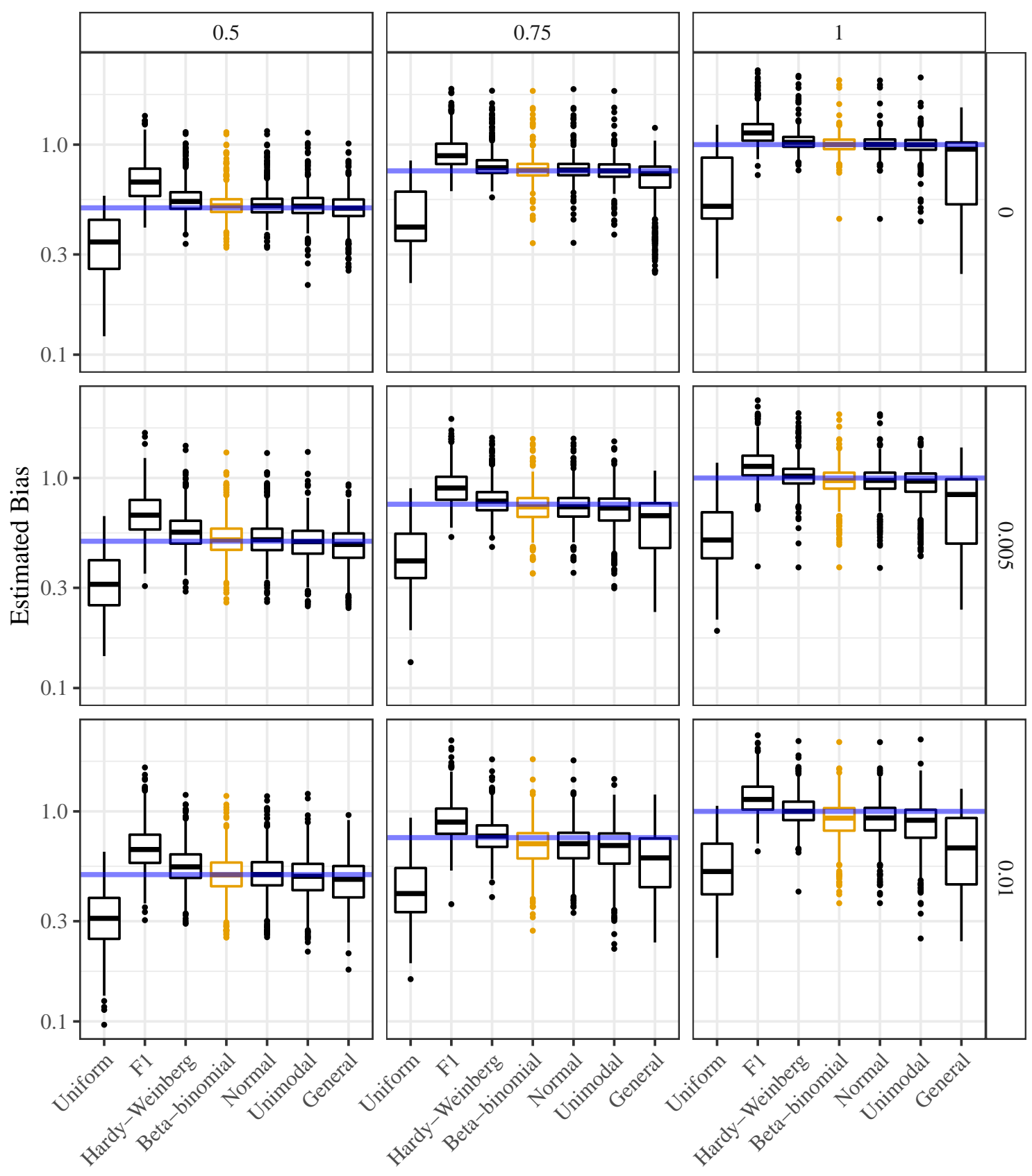

Assumed Prior

Figure S24: Estimated allele bias ( $y$-axis) stratified by the assumed class of prior distributions $(x-$ axis). The horizontal line is the actual allele bias. Column facets index varying levels of allele bias (with 1 indicating no bias) and row facets index varying levels of overdispersion (with 0 indicating no overdispersion). The genotypes were generated from a distribution designed to be favorable to the class of beta-binomial prior distributions (orange). 

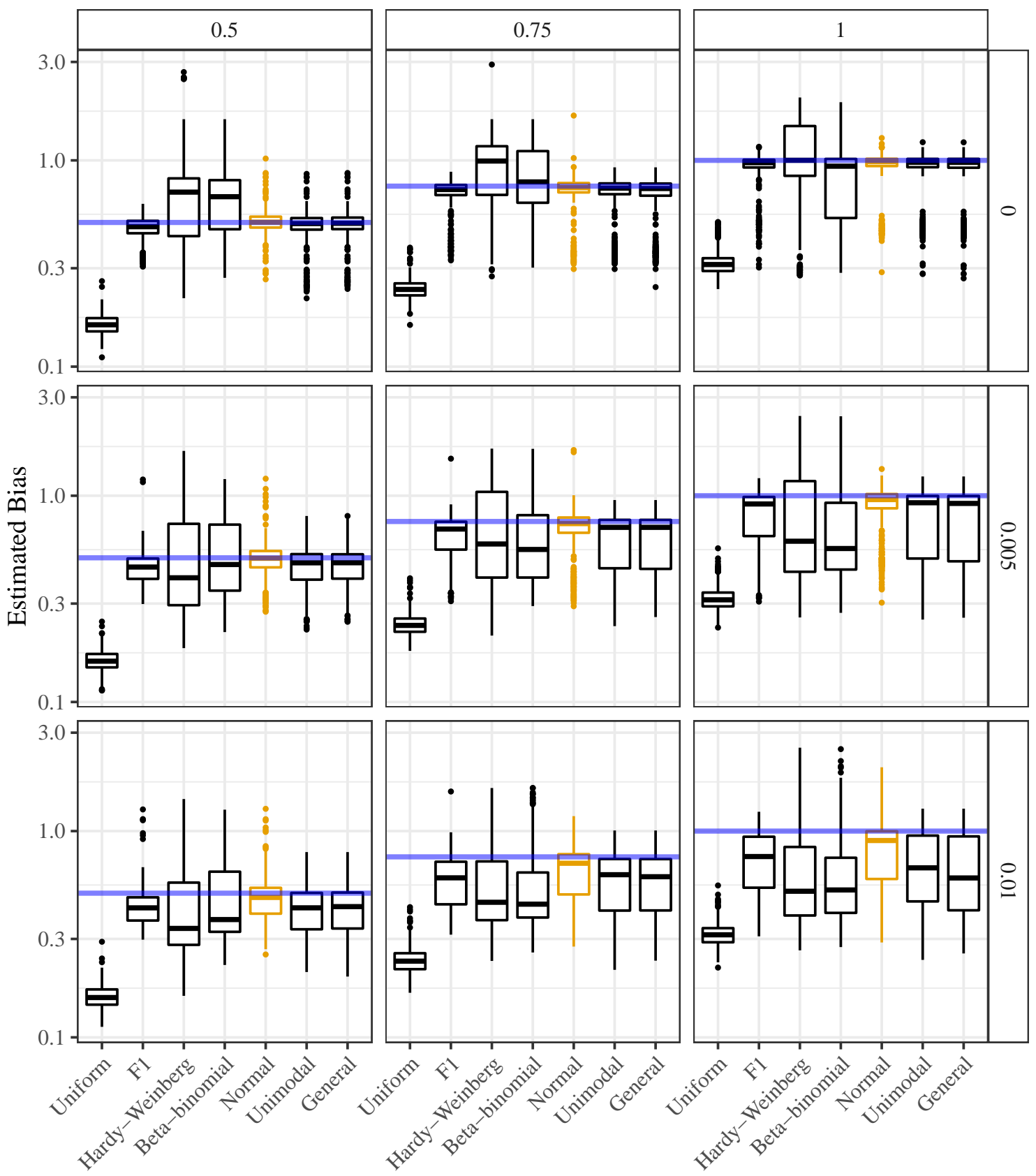

Assumed Prior

Figure S25: Estimated allele bias ( $y$-axis) stratified by the assumed class of prior distributions $(x-$ axis). The horizontal line is the actual allele bias. Column facets index varying levels of allele bias (with 1 indicating no bias) and row facets index varying levels of overdispersion (with 0 indicating no overdispersion). The genotypes were generated from a distribution designed to be favorable to the class of proportional normal prior distributions (orange). 

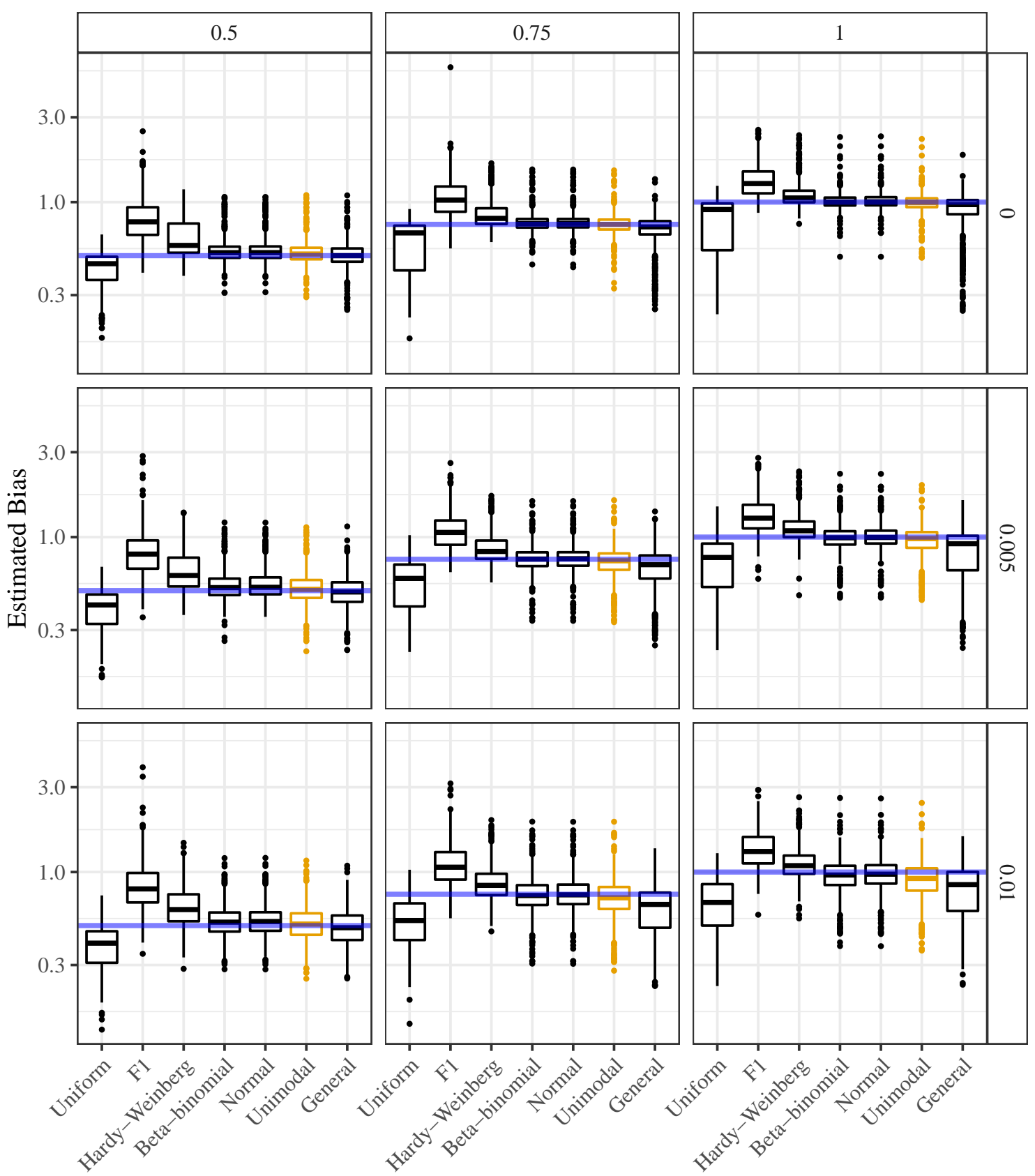

Assumed Prior

Figure S26: Estimated allele bias ( $y$-axis) stratified by the assumed class of prior distributions $(x-$ axis). The horizontal line is the actual allele bias. Column facets index varying levels of allele bias (with 1 indicating no bias) and row facets index varying levels of overdispersion (with 0 indicating no overdispersion). The genotypes were generated from a distribution designed to be favorable to the class of unimodal prior distributions (orange). 

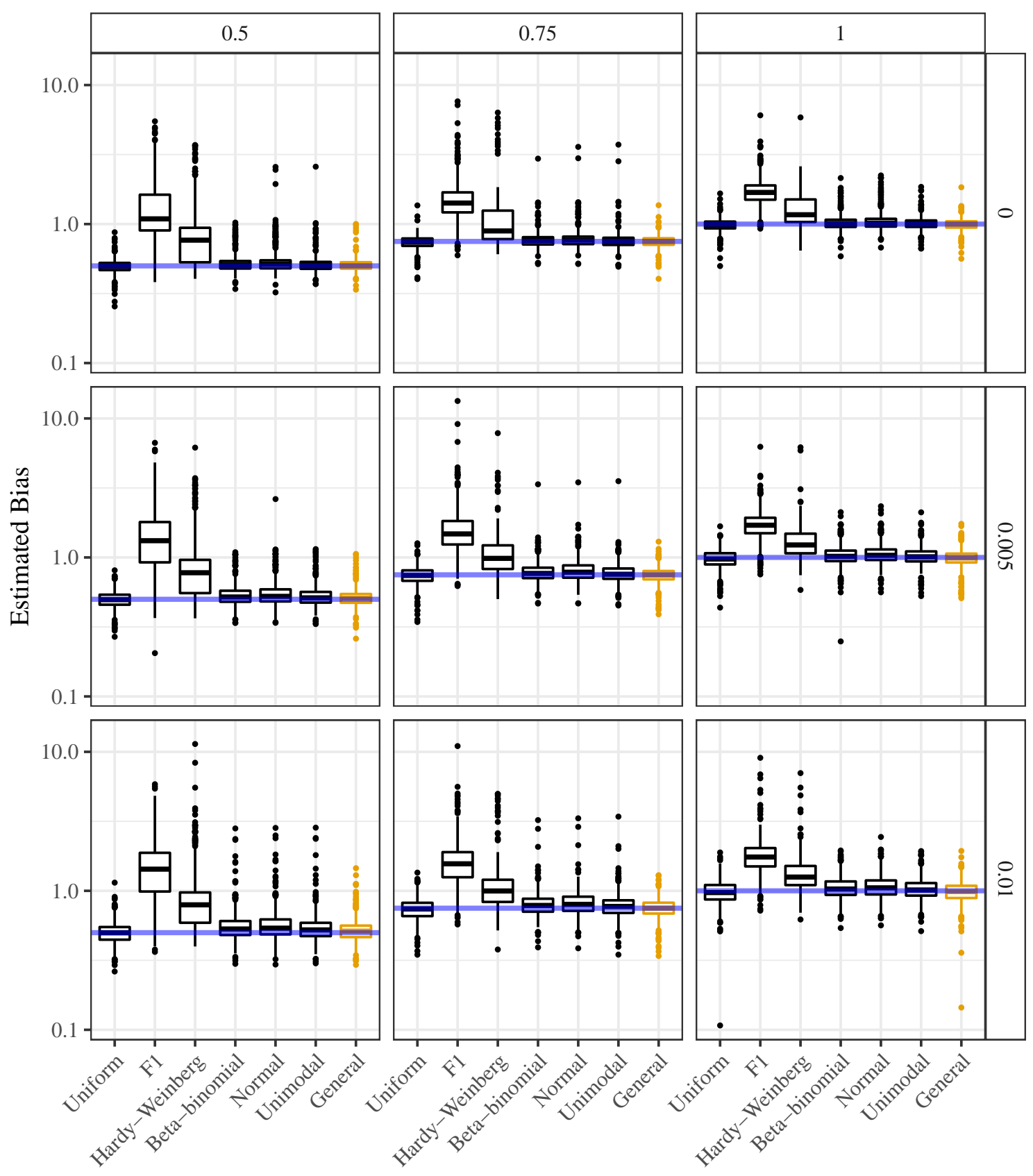

Assumed Prior

Figure S27: Estimated allele bias ( $y$-axis) stratified by the assumed class of prior distributions $(x$ axis). The horizontal line is the actual allele bias. Column facets index varying levels of allele bias (with 1 indicating no bias) and row facets index varying levels of overdispersion (with 0 indicating no overdispersion). The genotypes were generated from a distribution designed to be favorable to the class of general prior distributions (orange). 


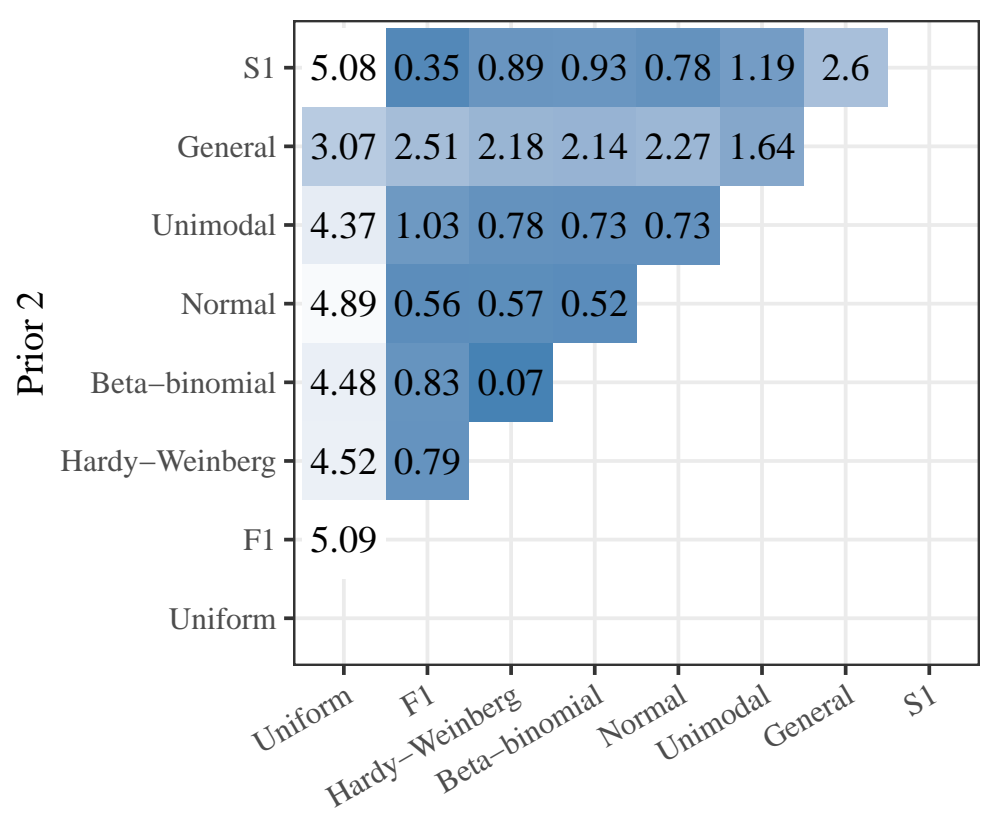

Prior 1

Figure S28: Mean Euclidean distance between posterior mean genotypes (color) resulting from two classes of prior distributions ( $x$ and $y$ axes). The "S1" prior is the true genotype distribution, so methods perform better when their posterior mean genotypes are closer to the S1 posterior mean genotypes (mean Euclidean distance is closer to 0). 


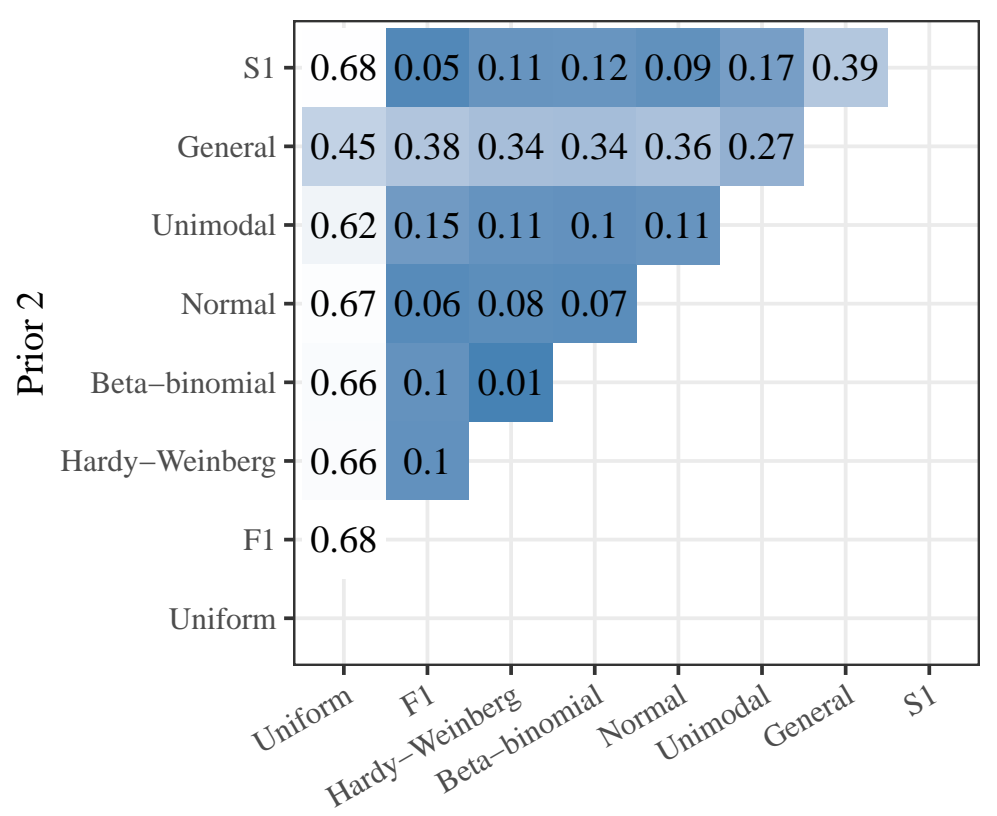

Prior 1

Figure S29: Mean proportion of posterior mode genotypes that are different (color). These posterior mode genotypes result from one of two classes of prior distributions ( $x$ and $y$ axes). The "S1" prior is the true genotype distribution, so methods perform better when their genotype estimates are closer to those resulting from an S1 prior (the mean proportions are closer to 0). 


\section{References}

Rodrigo R Amadeu, Luís Felipe Ventorim Ferrão, Ivone de Bem Oliveira, Juliana Benevenuto, Jeffrey B Endelman, and Patricio R Muñoz. Impact of dominance effects on autotetraploid genomic prediction. Crop Science, 2019. doi: 10.2135/cropsci2019.02.0138.

Nathan A. Baird, Paul D. Etter, Tressa S. Atwood, Mark C. Currey, Anthony L. Shiver, Zachary A. Lewis, Eric U. Selker, William A. Cresko, and Eric A. Johnson. Rapid SNP discovery and genetic mapping using sequenced RAD markers. PLOS ONE, 3(10):1-7, 10 2008. doi: 10.1371/journal.pone.0003376.

Juliana Benevenuto, Luís Felipe Ventorim Ferrão, Rodrigo R Amadeu, and Patricio Muñoz. How can a high-quality genome assembly help plant breeders? GigaScience, 8(6), 06 2019. ISSN 2047-217X. doi: 10.1093/gigascience/giz068.

Paul D Blischak, Laura S Kubatko, and Andrea D Wolfe. SNP genotyping and parameter estimation in polyploids using low-coverage sequencing data. Bioinformatics, 34(3):407-415, 2018. doi: 10.1093/bioinformatics/btx587.

Lindsay V. Clark, Alexander E. Lipka, and Erik J. Sacks. polyRAD: Genotype calling with uncertainty from sequencing data in polyploids and diploids. G3: Genes, Genomes, Genetics, 9(3):663-673, 2019. doi: $10.1534 / \mathrm{g} 3.118 .200913$.

J F Crow. Eighty years ago: the beginnings of population genetics. Genetics, 119(3):473-476, 1988. ISSN 0016-6731. URL https://www.genetics.org/content/119/3/473.

Ivone de Bem Oliveira, Marcio F. R. Resende, Luís Felipe Ventorim Ferrão, Rodrigo R. Amadeu, Jeffrey B. Endelman, Matias Kirst, Alexandre S. G. Coelho, and Patricio R. Muñoz. Genomic prediction of autotetraploids; influence of relationship matrices, allele dosage, and continuous genotyping calls in phenotype prediction. G3: Genes, Genomes, Genetics, 9(4):1189-1198, 2019. doi: 10.1534/g3.119.400059.

A. P. Dempster, N. M. Laird, and D. B. Rubin. Maximum likelihood from incomplete data via the EM algorithm. Journal of the Royal Statistical Society: Series B (Methodological), 39(1):1-22, 1977. doi: 10.1111/j.2517-6161.1977.tb01600.x.

Mark A DePristo, Eric Banks, Ryan Poplin, Kiran V Garimella, Jared R Maguire, Christopher Hartl, Anthony A Philippakis, Guillermo Del Angel, Manuel A Rivas, Matt Hanna, Aaron Mckenna, Tim Fennell, Andrew Kernytsky, Andrey Sivachenko, Kristian Cibulskis, Stacey Gabriel, David Altshuler, and Mark Daly. A framework for variation discovery and genotyping using next-generation DNA sequencing data. Nature genetics, 43(5):491-498, 2011. doi: 10.1038/ng.806.

Robert J. Elshire, Jeffrey C. Glaubitz, Qi Sun, Jesse A. Poland, Ken Kawamoto, Edward S. Buckler, and Sharon E. Mitchell. A robust, simple genotyping-by-sequencing (GBS) approach for high diversity species. PLOS ONE, 6(5):1-10, 05 2011. doi: 10.1371/journal.pone.0019379.

Jeffrey B. Endelman, Cari A. Schmitz Carley, Paul C. Bethke, Joseph J. Coombs, Mark E. Clough, Washington L. da Silva, Walter S. De Jong, David S. Douches, Curtis M. Frederick, Kathleen G. Haynes, David G. Holm, J. Creighton Miller, Patricio R. Muñoz, Felix M. Navarro, Richard G. Novy, Jiwan P. Palta, Gregory A. Porter, Kyle T. Rak, Vidyasagar R. Sathuvalli, Asunta L. Thompson, and G. Craig Yencho. Genetic variance partitioning and genome-wide prediction with allele dosage information in autotetraploid potato. Genetics, 209(1):77-87, 2018. ISSN 0016-6731. doi: 10.1534/genetics.118.300685.

Luís Felipe Ventorim Ferrão, Juliana Benevenuto, Ivone de Bem Oliveira, Catherine Cellon, James Olmstead, Matias Kirst, Marcio F. R. Resende, and Patricio Muñoz. Insights into the genetic basis of blueberry fruitrelated traits using diploid and polyploid models in a GWAS context. Frontiers in Ecology and Evolution, 6:107, 2018. ISSN 2296-701X. doi: 10.3389/fevo.2018.00107.

Rebecca Caroline Ulbricht Ferreira, Letícia Aparecida de Castro Lara, Lucimara Chiari, Sanzio Carvalho Lima Barrios, Cacilda Borges do Valle, José Raul Valério, Fabrícia Zimermann Vilela Torres, Antonio Augusto Franco Garcia, and Anete Pereira de Souza. Genetic mapping with allele dosage information in tetraploid Urochloa decumbens (stapf) r. d. webster reveals insights into spittlebug (Notozulia entreriana berg) resistance. Frontiers in Plant Science, 10:92, 2019. ISSN 1664-462X. doi: 10.3389/fpls.2019.00092.

Anqi Fu, Balasubramanian Narasimhan, and Stephen Boyd. CVXR: An R package for disciplined convex optimization. arXiv preprint arXiv:1711.07582, 2017. URL https://arxiv.org/abs/1711.07582. 
David Gerard, Luís Felipe Ventorim Ferrão, Antonio Augusto Franco Garcia, and Matthew Stephens. Genotyping polyploids from messy sequencing data. Genetics, 210(3):789-807, 2018. ISSN 0016-6731. doi: 10.1534/genetics.118.301468.

Letícia Aparecida de Castro Lara, Mateus F. Santos, Liana Jank, Lucimara Chiari, Mariane de M. Vilela, Rodrigo R. Amadeu, Jhonathan P. R. dos Santos, Guilherme da S. Pereira, Zhao-Bang Zeng, and Antonio Augusto F. Garcia. Genomic selection with allele dosage in Panicum maximum jacq. G3: Genes, Genomes, Genetics, 9(8):2463-2475, 2019. doi: 10.1534/g3.118.200986.

Heng Li. A statistical framework for SNP calling, mutation discovery, association mapping and population genetical parameter estimation from sequencing data. Bioinformatics, 27(21):2987, 2011. doi: 10.1093/bioinformatics/btr509.

E. R. Martin, D. D. Kinnamon, M. A. Schmidt, E. H. Powell, S. Zuchner, and R. W. Morris. SeqEM: an adaptive genotype-calling approach for next-generation sequencing studies. Bioinformatics, 26(22): 2803-2810, 2010. doi: 10.1093/bioinformatics/btq526.

Takahiro Maruki and Michael Lynch. Genotype calling from population-genomic sequencing data. G3: Genes, Genomes, Genetics, 7(5):1393-1404, 2017. doi: 10.1534/g3.117.039008.

Aaron McKenna, Matthew Hanna, Eric Banks, Andrey Sivachenko, Kristian Cibulskis, Andrew Kernytsky, Kiran Garimella, David Altshuler, Stacey Gabriel, Mark Daly, and Mark DePristo. The genome analysis toolkit: a MapReduce framework for analyzing next-generation DNA sequencing data. Genome research, 20(9):1297-1303, 2010. doi: 10.1101/gr.107524.110.

R Core Team. R: A Language and Environment for Statistical Computing. R Foundation for Statistical Computing, Vienna, Austria, 2019. URL https://www.R-project.org/.

Umesh R Rosyara, Walter S De Jong, David S Douches, and Jeffrey B Endelman. Software for genome-wide association studies in autopolyploids and its application to potato. The Plant Genome, 9(2), 2016. doi: 10.3835/plantgenome2015.08.0037.

Oliver Serang, Marcelo Mollinari, and Antonio Augusto Franco Garcia. Efficient exact maximum a posteriori computation for Bayesian SNP genotyping in polyploids. PLOS ONE, 7(2):1-13, 02 2012. doi: 10.1371/journal.pone.0030906.

Kenta Shirasawa, Masaru Tanaka, Yasuhiro Takahata, Daifu Ma, Qinghe Cao, Qingchang Liu, Hong Zhai, Sang-Soo Kwak, Jae Cheol Jeong, Ung-Han Yoon, Hyeong-Un Lee, Hideki Hirakawa, and Sahiko Isobe. A high-density SNP genetic map consisting of a complete set of homologous groups in autohexaploid sweetpotato (Ipomoea batatas). Scientific Reports, 7, 2017. doi: 10.1038/srep44207.

Douglas E Soltis, Clayton J Visger, and Pamela S Soltis. The polyploidy revolution then. . .and now: Stebbins revisited. American Journal of Botany, 101(7):1057-1078, 2014. doi: 10.3732/ajb.1400178.

Pamela S. Soltis and Douglas E. Soltis. The role of genetic and genomic attributes in the success of polyploids. Proceedings of the National Academy of Sciences, 97(13):7051-7057, 2000. doi: 10.1073/pnas.97.13.7051.

Matthew Stephens. False discovery rates: a new deal. Biostatistics, 18(2):275-294, 10 2016. ISSN 1465-4644. doi: 10.1093/biostatistics/kxw041.

M. Stift, R. Reeve, and P. H. Van Tienderen. Inheritance in tetraploid yeast revisited: segregation patterns and statistical power under different inheritance models. Journal of Evolutionary Biology, 23(7):15701578, 2010. doi: 10.1111/j.1420-9101.2010.02012.x.

Joshua A Udall and Jonathan F Wendel. Polyploidy and crop improvement. Crop Science, 46(Supplement_1): S-3, 2006. doi: 10.2135/cropsci2006.07.0489tpg.

Jan G. A. M. L. Uitdewilligen, Anne-Marie A. Wolters, Bjorn B. D'hoop, Theo J. A. Borm, Richard G. F. Visser, and Herman J. van Eck. A next-generation sequencing method for genotyping-by-sequencing of highly heterozygous autotetraploid potato. PLOS ONE, 8(5):1-14, 05 2013. doi: 10.1371/journal.pone.0062355.

Roeland E. Voorrips and Chris A. Maliepaard. The simulation of meiosis in diploid and tetraploid organisms using various genetic models. BMC Bioinformatics, 13(1):248, Sep 2012. ISSN 1471-2105. doi: 10.1186/1471-2105-13-248. 
bioRxiv preprint doi: https://doi.org/10.1101/751784; this version posted August 31, 2019. The copyright holder for this preprint (which was not certified by peer review) is the author/funder, who has granted bioRxiv a license to display the preprint in perpetuity. It is made available under aCC-BY-NC-ND 4.0 International license.

Roeland E. Voorrips, Gerrit Gort, and Ben Vosman. Genotype calling in tetraploid species from biallelic marker data using mixture models. BMC Bioinformatics, 12(1):172, 2011. ISSN 1471-2105. doi: 10.1186/1471-2105-12-172.

Xiaoquan Wen and Matthew Stephens. Using linear predictors to impute allele frequencies from summary or pooled genotype data. The annals of applied statistics, 4(3):1158-1182, 2010. ISSN 1932-6157. doi: 10.1214/10-aoas338.

Hadley Wickham. ggplot2: Elegant Graphics for Data Analysis. Springer-Verlag New York, 2016. ISBN 978-3-319-24277-4. URL https://ggplot2.tidyverse.org. 